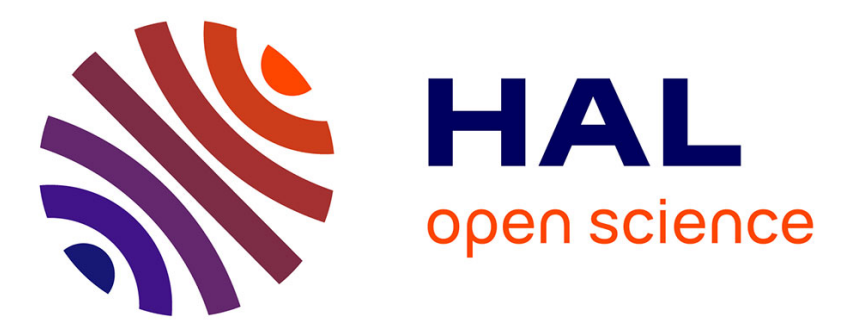

\title{
Wind-Tunnel and CFD Investigations Focused on Transition and Performance Predictions of Laminar Wings
}

David Hue, Olivier Vermeersch, Jérémy Duchemin, Olivier Colin, Dac Tran

\section{- To cite this version:}

David Hue, Olivier Vermeersch, Jérémy Duchemin, Olivier Colin, Dac Tran. Wind-Tunnel and CFD Investigations Focused on Transition and Performance Predictions of Laminar Wings. AIAA Journal, 2017, 56 (1), pp.132-145. 10.2514/1.J056088 . hal-01727710

\author{
HAL Id: hal-01727710 \\ https://hal.science/hal-01727710
}

Submitted on 10 Oct 2018

HAL is a multi-disciplinary open access archive for the deposit and dissemination of scientific research documents, whether they are published or not. The documents may come from teaching and research institutions in France or abroad, or from public or private research centers.
L'archive ouverte pluridisciplinaire HAL, est destinée au dépôt et à la diffusion de documents scientifiques de niveau recherche, publiés ou non, émanant des établissements d'enseignement et de recherche français ou étrangers, des laboratoires publics ou privés. 


\title{
Wind-Tunnel and CFD Investigations Focused on Transition and Performance Predictions of Laminar Wings
}

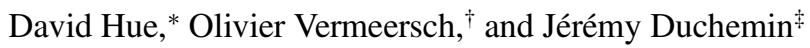 \\ ONERA-The French Aerospace Lab, 92190 Meudon, France \\ and \\ Olivier Colin $\underline{\S}$ and Dac Tran \\ Dassault Aviation, 92210 Saint-Cloud, France
}

\begin{abstract}
DOI: $\underline{10.2514 / 1 . J 056088}$
This paper is a continuation of the paper recently published by Hue et al. ("Experimental and Numerical Methods for Transition and Drag Predictions of Laminar Airfoils," AIAA Journal, Vol. 53, No. 9, Sept. 2015, pp. 2694-2712) that focused on transition and drag predictions of laminar airfoils. The extension of such studies to three-dimensional configurations representative of modern civil aircraft is a further step toward the implementation of natural laminarflow technologies. The present work, therefore, focuses on validating the laminar design of a low-swept wing for business jet applications. In 2015, an experimental campaign was carried out in the European Transonic Windtunnel, and included laminar/turbulent transition measurements with temperature-sensitive paint at Mach and Reynolds numbers typical of cruise flight conditions. Subsequently, fluid dynamics computations were performed on this aircraft geometry either with a Reynolds-averaged Navier-Stokes solver using both Tollmien-Schlichting and crossflow transition criteria, or with a boundary-layer code using either database methods for transition location or exact stability analyses. In this paper, experimental and numerical transition predictions are compared for three representative cases corresponding to different angles of attack. The agreement that is achieved is satisfactory, and extended regions of laminar flow are observed on the wing at cruise lift levels. In these conditions, the drag reduction can account for 10-15\% of the aircraft drag.
\end{abstract}

\section{Nomenclature}

$C D_{f} \quad=$ friction-drag coefficient

$C D_{f f}=$ far-field drag coefficient

$C D_{i} \quad=$ lift-induced drag coefficient

$C D_{\mathrm{nf}}=$ near-field drag coefficient

$C D_{p} \quad=$ pressure drag coefficient

$C D_{\text {sp }}=$ spurious drag coefficient

$C D_{v}=$ viscous drag coefficient

$C D_{\text {vp }}=$ viscous-pressure drag coefficient

$C D_{w}=$ wave-drag coefficient

$C_{L}=$ lift coefficient

$c \quad=$ wing chord

$f \quad=$ frequency of boundary-layer instability

$K_{p} \quad=$ pressure coefficient

$\mathrm{Ma}=$ Mach number

$N_{T} \quad=\quad$ value of factor $N$ at transition onset

$P_{i} \quad=\quad$ stagnation pressure

Re $\quad=$ Reynolds number

$s \quad=$ semispan

$T_{i} \quad=\quad$ stagnation temperature

$T u=$ turbulence level

$u, v, w=x, y, z$ velocity components

$Y^{+} \quad=$ normalized first cell height

$\alpha \quad=$ angle of attack $\begin{array}{ll}\eta & =\text { fraction of wingspan } \\ \rho & =\text { density }\end{array}$

\section{Introduction}

TUDIES focused on the issue of laminar flow over airfoils and wings have been carried out for several decades now [1 $\underline{1} \underline{3}]$. In the context of rising fuel costs and strict environmental requirements, investing in the reduction of friction drag, the largest component of drag for civil aircraft, is logical. Along this path, decisive steps have been accomplished, from the NACA 6-series and natural laminar flow (NLF) subsonic airfoils [4] to the development of high-speed and polyvalent laminar profiles [5-7]. Recently, in the 2000s, within the European project Telfona [8,9], an aircraft model named Pathfinder was designed, built, and tested with the aim of obtaining NLF over the wings for transonic conditions with high Reynolds number in the European Transonic Windtunnel (ETW).

In direct continuation of this project, further efforts from the European Joint Technology Initiatives (JTI) Clean Sky, Smart Fixed Wing Aircraft (SFWA), started in 2010, have focused on natural laminar design for innovative next-generation aircraft. In this context, Dassault Aviation and ONERA-The French Aerospace Lab first carried out studies on two-dimensional (2-D) transonic laminar airfoils that included a test campaign held at the ONERA-The French Aerospace Lab S2MA wind tunnel in 2012. The objectives of these experiments were to evaluate the laminar behavior of these airfoils in transonic conditions, study laminar buffet, and acquire a large and detailed database for comparison purposes. The ONERA-The French Aerospace Lab computational fluid dynamics (CFD) methods were then validated for this 2-D case both in terms of transition-location prediction and relative-performance-gain assessment. A paper presenting the results of this work was published in the AIAA Journal in 2015 [10].

Still within the JTI-SFWA project, a full aircraft called low-sweep business jet (LSBJ) was designed by Dassault Aviation. It is fitted with laminar transonic wings and innovative concepts, which ensure a low environmental impact. A scale model was manufactured, and then tested at ETW in 2015 during a campaign that included cryogenic temperature-sensitive-paint (TSP) methods (cryoTSP) to 
locate the transition position, static and unsteady pressure sensors, and force measurements (balance). Moreover, a great number of CFD computations were performed by ONERA-The French Aerospace Lab on this three-dimensional (3-D) configuration: first, with a Reynolds-averaged Navier-Stokes (RANS) solver using transition criteria, and then with a boundary-layer code involving a database method for transition prediction. Some of these results have been validated with exact stability computations. These experimental and numerical studies based on the laminar LSBJ aircraft are the topic of the present publication.

In contrast with the 2-D study presented in [10], this paper considers a 3-D configuration, for which transition to turbulence will be driven not only by the amplification of Tollmien-Schlichting (TS) instabilities, but also by the crossflow (CF) mechanisms.

At first, the paper will describe the ETW experimental setup, LSBJ model, measurement techniques, and test matrix. The numerical features and methods will then be explained: first, details about the transition prediction will be given (TS and CF criteria, as well as the boundary-layer and stability approaches), and then the overset grids, RANS solver elsA, and far-field drag-extraction code ffd 72 that were used in this study will be presented. In the subsequent section, experimental and numerical results will be compared. First, the focus will be on transition prediction: several cases of interest will be highlighted (cruise conditions, low and high angles of attack, specific transition mechanisms, etc.), and then aircraft performance will be analyzed, and benefits in lift and drag due to extended laminar boundary layers will be evaluated. At last, general conclusions will be drawn.

\section{Description of the Experiments}

\section{A. Wind-Tunnel Facility}

The test campaign presented in this study was carried out in October 2015 at ETW (High-Speed Wind-Tunnel Test of a LaminarConfiguration Bizjet project). ETW is a cryogenic pressurized transonic wind tunnel located in Cologne, Germany. It is a closedloop circuit facility whose test section is $2 \mathrm{~m}$ high and $2.4 \mathrm{~m}$ wide. The inflow Mach number ranges between 0.15 and 1.35. The gas flow consists of pure nitrogen allowing low stagnation temperature $T_{i}$ from 110 to $313 \mathrm{~K}$. The stagnation pressure $P_{i}$ can be adjusted independently from the temperature from 1.15 to 4.5 bars. This allows Reynolds numbers per meter of more than 60 million to be achieved.

One of the main issues of studies focused on laminar-to-turbulent transition in wind tunnels is the level of disturbances in the test section. Disturbance levels are quantified using the turbulence-level parameter $T u$ : in particular, as $T u$ increases, transition occurs closer to the leading edge [11]. Flow quality was investigated [12] in the pilot facility of ETW (PETW) representing a scaled version of ETW (1:8.8), but covering the same operational range (total pressures and temperatures, as well as Mach numbers). Careful analyses of hotwire signal for a wide range of conditions have shown that the turbulence level varied between $0.05 \%<T u<0.6 \%$ depending on the inflow Mach number (with a small dependence to temperature and density).

From these measurements, it is possible to calculate an $N$ factor corresponding to transition onset. The $N$ factor, defined as the integration of amplification rates, describes the amplification of disturbances propagating inside the boundary layer [13,14]. Because the initial amplitude $A_{0}$ of the boundary-layer instabilities depends on the external disturbance level, the stronger the turbulence level is, the smaller the critical $N$ factor $N_{T}$, corresponding to transition onset, will be. Gathering a large number of wind-tunnel transition-location measurements, Mack [15] established for 2-D configurations (TS-induced transition) a correlation between the $N$ factor at transition and external turbulence level:

$$
N_{T}=-8.43-2.4 \times \ln (T u)
$$

This provides a large range of critical $N$ factor values from 4 to 10 for the PETW. However, restricting the integration of hot-wire power spectral density between 10 and $40 \mathrm{kHz}$ (which covers the unstable frequency range of TS instabilities) gives a critical factor $N_{T}$ of 8 . These values measured in PETW are well suited for laminar-flow studies, and the order of magnitude should be the same in the full-size wind-tunnel ETW. Indeed, as it will be demonstrated later, $N_{T}=8$ allows a good agreement between $e^{N}$ transition prediction and measurements for the TS-induced transition.

\section{B. Model and Measurement Techniques}

The LSBJ model (1:14.7) has been designed by Dassault Aviation and manufactured by French companies (Vallet, CMA, and TECSERM) in the framework of the IDAMME2 project. The model was mounted by a $\mathrm{Z}$ sting in the test section, as represented in Fig. 1 . It has a full span of about $1.5 \mathrm{~m}$ and a mean aerodynamic chord of approximately $0.15 \mathrm{~m}$. To measure the chordwise evolution of pressure distribution, the wing was equipped with 113 wall static pressure taps: 80 located on the suction side and 33 on the pressure side. Pressure taps had a diameter of $200 \mu \mathrm{m}$. The model had a removable horizontal tailplane with remote motorization, as well as interchangeable wing trailing edges to analyze the camber effect on overall aircraft performance. Aerodynamic forces and moments were measured by a six-component internal balance inside the model.

During the campaign, laminar/turbulent transition locations were measured by the DLR, German Aerospace Center and ETW teams using cryoTSP $[16,17]$ on the model's starboard wing. Pressure and suction sides were both equipped with three inserts filled with cryoTSP and covering an area between 15 and $65 \%$ of the chord. Because heat exchange is greater in turbulent flows (compared to laminar flows), a significant wall-temperature difference on either side of the transition line can be recorded using cryoTSP. An example of cryoTSP visualization is represented in Fig. 2a on the pressure side of the wing. On this figure, transition location, which separates the laminar area (dark) from the turbulent area (bright), can be estimated visually. The chordwise evolution of the wall temperature (in gray levels) was extracted in Fig. $2 \mathrm{~b}$ at two spanwise stations $y / s=39 \%$ (full line) and 59\% (dashed line). Wall temperature exhibits a sudden rise that extends on several percent of chord from $T_{w} \approx 9975 \mathrm{Gl}$ (laminar value) to $T_{w} \approx 10075 \mathrm{Gl}$ (turbulent value). Considering the temperature evolution in the $39 \%$ section, transition starts at $x / c=25 \%$ and ends at $34 \%$ of chord. This shows that transition onset is complex and not restricted to a single point, but is a more or less an extended area. Indeed, physically laminar zones coexist with the first turbulent spots. This means that the specification of experimental transition location is not straightforward. In the following analyses, the measured transition location will be defined as the abscissa where temperature increase starts $\left(x_{T} / c=25 \%\right.$ for $y / s=39 \%$ in Fig. 2b)

As it can be observed in Fig. 2a, the TSP visualization presents turbulent wedges, which make it even more difficult to physically interpret the transition process as well as determine the transition

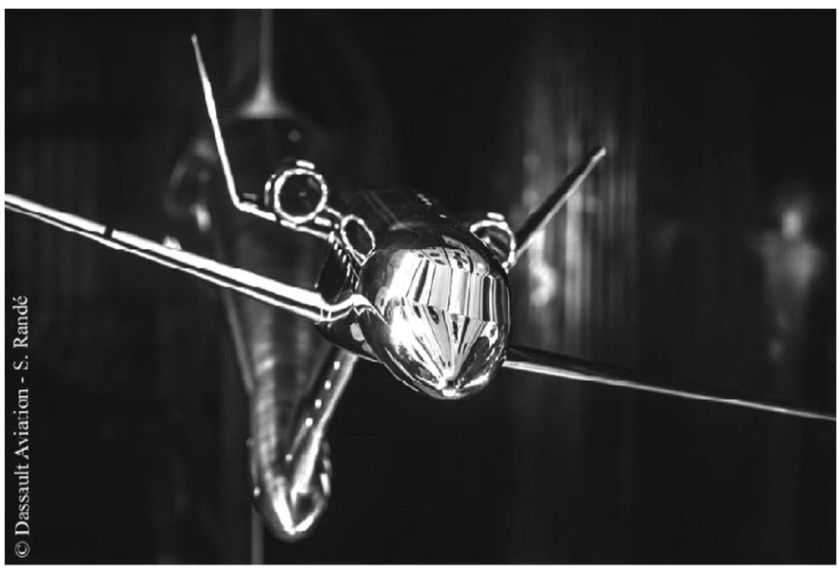

Fig. 1 LSBJ model in ETW test section. 

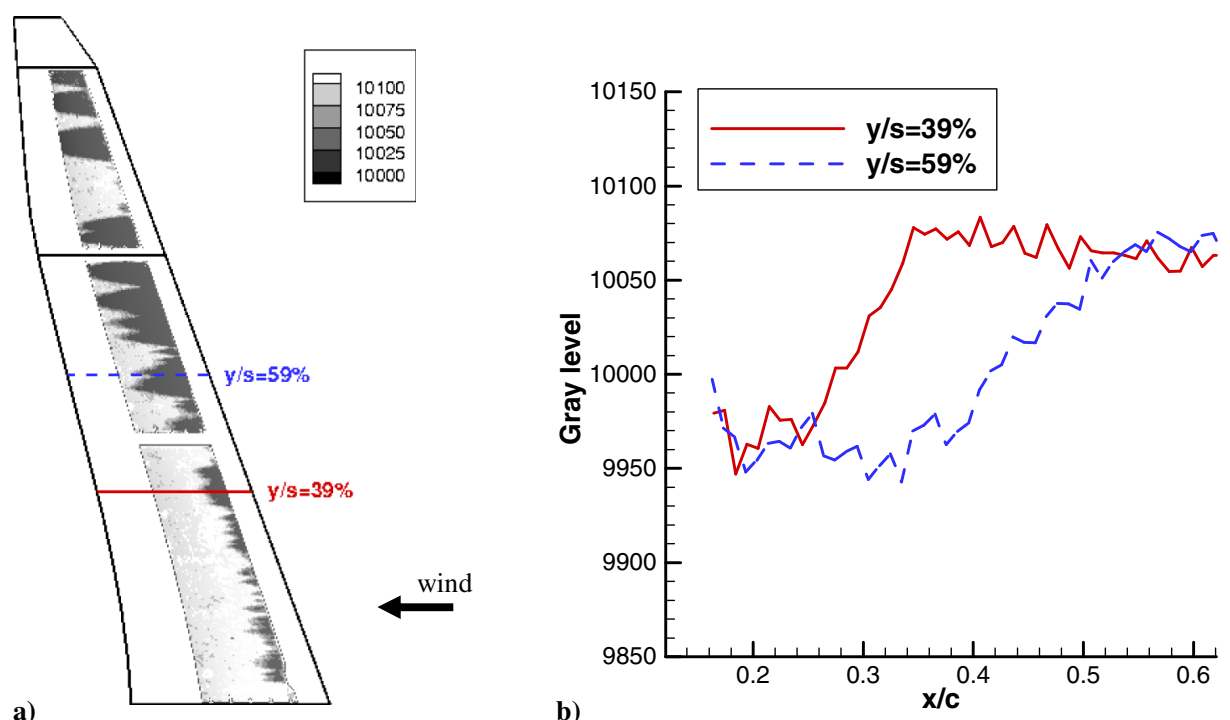

Fig. 2 Example of cryoTSP visualization and chordwise evolution of wall temperature for two spanwise sections.

location. These undesired wedges are due to local surface roughness and/or imperfect junction between the wing surface and the cryoTSP insert. Additionally, it should be mentioned that, as TSP highlights wall-temperature gradient, it will be sensitive to shock wave ( $\Delta T>0$, if the flow is warmer than the model) or boundary-layer separation $(\Delta T<0)$.

\section{Test Matrix}

During the experimental campaign, a great number of conditions were tested with a Mach number ranging from 0.7 to 0.82 for different settings of the tailplane and the wing camber. In parallel, the stagnation temperature and pressure were varied to study the influence of Reynolds number with the same wing deformation. Table 1 shows only a part of the whole test matrix corresponding to the particular cases that will make up the main focus of the present paper, in terms of laminarto-turbulent transition and performance prediction. To quantify the impact of laminar flow on aerodynamic coefficients, the transition location was either triggered or not. Transition triggering was done on both sides of the wing. For the suction side, the transition line was located at $5 \%$ chord at the root and at $15 \%$ chord at the tip, whereas for the pressure side, $8 \%$ chord at the root and $12 \%$ at the tip. It was managed using Cad Cut devices whose heights were close to the boundary-layer displacement thickness $(\approx 80 \mu \mathrm{m})$.

\section{Numerical Methods}

In this section, after a brief overview on the transition mechanisms in a 3-D boundary layer, all the numerical approaches used in this study will be presented. First, the focus will be on transitionprediction methods. They will be introduced in order of accuracy, starting from the most accurate and CPU-time-consuming method to the simplest one. Secondly, the computational grids and RANS solver will be presented. Finally, a far-field drag-extraction code that is used for performance assessment will be described.

\section{A. Transition Mechanisms}

On a swept wing, for moderate turbulence levels, such as those found in flight conditions, the transition to turbulence may be driven by two kinds of instabilities, namely, the TS instability and the $\mathrm{CF}$ instability. These instabilities are called modal instabilities because they are related to boundary-layer eigenmodes. The development of both TS and CF modes follows three main steps. The first one, called receptivity, takes place close to the leading edge and describes how external disturbances enter the boundary layer to excite its eigenmodes. Some of these modes are then amplified in the streamwise direction through the second phase called linear amplification. The third and last step, because mode amplitudes have become large, nonlinear interactions rapidly lead to turbulent-spot formation.

In 2-D configurations, the boundary-layer instabilities are the TS waves. They are generated inside the boundary layer by freestream turbulence (both velocity $u^{\prime}$ and noise/pressure $p^{\prime}$ fluctuations), and they are all the more amplified when the external velocity gradient is negative, that is, the pressure gradient is positive: $d P / d x>0$.

In 3-D cases, such as a swept wing or a fuselage, the boundarylayer mean-velocity profile has two components in a plane parallel to the wall: a streamwise component and a transverse (or $\mathrm{CF}$ ) component.

In the streamwise-velocity component, fluctuations similar to the 2-D TS waves evolve. The transverse-velocity profile is characterized by an inflection point, which is responsible for the generation of $\mathrm{CF}$ instabilities. These $\mathrm{CF}$ vortices will be amplified in a positive freestream velocity gradient (i.e., a negative pressure gradient: $d P / d x<0$ ). Both stationary (zero frequency, $f=0$ ) and traveling $(f \neq 0)$ disturbances may exist in the boundary layer.

Considering chordwise pressure distribution across a conventional airfoil, the CF instabilities are supposed to be amplified in the accelerated-flow region near the leading edge, whereas TS waves will be amplified downstream in the decelerated-flow region. Therefore, the NLF design consists in optimizing pressure distribution along the airfoil with a strongly accelerated zone as short as possible and downstream the preservation as far as possible of a mild negative pressure gradient.

As TS and CF instabilities are related to boundary-layer eigenmodes, in the linear phase, the same stability theory is applicable to both.

Table 1 Part of the test matrix

\begin{tabular}{|c|c|c|c|c|c|}
\hline $\begin{array}{l}\text { Mach } \\
\text { number } M a\end{array}$ & $\begin{array}{c}\text { Stagnation } \\
\text { pressure } P_{i}, \text { bar }\end{array}$ & $\begin{array}{c}\text { Stagnation } \\
\text { temperature } T_{i}, \mathrm{~K}\end{array}$ & $\begin{array}{c}\text { Chord Reynolds } \\
\text { number } \operatorname{Re}\left(\times 10^{6}\right)\end{array}$ & Lift range $C_{L}$ & $\begin{array}{c}\text { Wing-transition } \\
\text { triggering }\end{array}$ \\
\hline 0.75 & 1.38 & 120 & 9.75 & $0.20-1.00$ & On + off \\
\hline 0.78 & 1.35 & 120 & 9.75 & $0.20-1.00$ & On + off \\
\hline
\end{tabular}




\section{B. Linear-Stability Theory for the Laminar Boundary Layer}

The linear-stability theory aims at computing the exponential growth of modal CF and TS instabilities. Disturbances are introduced in the form of waves in the compressible Navier-Stokes equations:

$q^{\prime}(x, y, z, t)=\hat{q}(z) \times \exp \left(-\alpha_{i} \cdot x\right) \times \exp \left[i \cdot\left(\alpha_{r} \cdot x+\beta \cdot y-\omega \cdot t\right)\right]$

for which $q^{\prime}$ is a velocity $\left(u^{\prime}, v^{\prime}, w^{\prime}\right)$, pressure $\left(p^{\prime}\right)$, or temperature fluctuation, and $\hat{q}$ is its corresponding amplitude function.

$\alpha=\alpha_{r}+i \cdot \alpha_{i}$ and $\beta$ are the streamwise ( $x$ direction) and spanwise ( $y$ direction) wave numbers, respectively, and $\omega=2 \pi \times f$ the angular frequency. Dealing with spatial amplification, the boundary-layer stability depends on the sign of $\alpha_{i}$ : unstable (respectively stable) if $\alpha_{i}$ is negative (respectively positive). NavierStokes equations are then linearized assuming that fluctuations are weak compared to mean-flow quantities, which leads to a system of ordinary differential equations for the amplitude functions (eigenvalue problem). This system can be reduced to a single fourthorder differential equation for the wall-normal velocity fluctuation $w^{\prime}$ well-known as the Orr-Sommerfeld equation (established in 1908) completed by the Squire equation (1933) for the wall-normal vorticity, whose solution provides boundary-layer eigenmodes. It is convenient to introduce the angle between the external streamline and the wave vector: $\Psi=\tan ^{-1}\left(\beta / \alpha_{r}\right)$.

The $N$ factor is thus defined as the integration of the amplification rate $\alpha_{i}$ along the external streamline, and describes the total amplification of the boundary-layer instability.

$$
N(x, \psi, f)=-\int_{x_{0}}^{x} \alpha_{i}(\xi, \psi, f) \cdot \mathrm{d} \xi
$$

If the initial amplitude $A_{0}$ of a given instability [i.e. $(\Psi, f)$ fixed] is introduced at a position $x_{0}$, the $N$ factor induced:

$$
\frac{A(x)}{A_{0}}=e^{N(x)}
$$

Transition prediction based on the linear-stability theory relies on the hypothesis that transition will be triggered if the $N$ factor [Eq. (3)] exceeds a given value i.e., if the boundary-layer instability is sufficiently amplified [Eq. (4)]\}.

In this paper, the TS and CF instabilities are studied separately, such that distinct $N$ factors are defined for each of them. The TS disturbances will be computed for several values of wave-vector direction: 2 -D TS waves ( $\Psi=0 \mathrm{deg}$ ) as well as oblique TS waves ( $\Psi=20,40$, and $60 \mathrm{deg}$ ). The $N$ factor for TS disturbances will correspond to the most amplified direction and frequency. It gives

$$
N_{\mathrm{TS}}(x)=\max _{f}\left\{\max _{0 \leq \psi \leq 60 \mathrm{deg}}\left[-\int_{x_{0}}^{x} \alpha_{i}(\xi, \psi, f) \cdot \mathrm{d} \xi\right]\right\}
$$

and the corresponding critical $N$-factor value for the TS-induced transition is set to $N_{T, \mathrm{TS}}=8$. that

$\mathrm{CF}$ disturbances are restricted to their stationary component, such

$$
N_{\mathrm{CF} 0}(x)=\int_{x_{0}}^{x} \max _{85<\psi<89}\left[-\alpha_{i}(\xi, \psi, f=0)\right] \cdot \mathrm{d} \xi
$$

As for TS instabilities, the critical $N$ factor for the CF-induced transition is fixed at $N_{T, \mathrm{CF}_{0}}=8$. It should be mentioned that, a priori, these critical values should be distinct, because TS and CF disturbances are not generated inside the boundary layer by the same receptivity mechanisms, and do not interfere with each other during the amplification phase. These critical values for transition onset are in good agreement with the ones given in [8].

As mentioned before, both stationary and traveling CF instabilities may exist inside the boundary layer. Some stability analyses of traveling CF have been undertaken (not presented here for the sake of simplicity). Unstable CF modes are characterized by frequencies around $10 \mathrm{kHz}$, and undergo strong amplification with $10<N<12$ at the transition onset. However, not a single critical $N$-factor value was able to correlate the measured transition locations contrary to stationary mode for which $N_{T}=8$ matched with most of the measurements. Additionally, as mentioned in Sec. II.A, above a given frequency in the order of $10 \mathrm{kHz}$, the power spectral density exhibits very low fluctuation level in external flow, meaning that the forcing of traveling crossflow by freestream turbulence is weak.

The linear-stability theory combined with the $e^{N}$ approach is a powerful tool for transition prediction. Nonetheless, it has some drawbacks. Firstly, as the stability of the boundary-layer velocity profiles is directly analyzed, they must be accurate with at least 50 points inside the boundary layer. Secondly, it requires solving an eigenvalue problem at each station of the instability path, which has to be done for each physical disturbance, meaning a single frequency $f$ and spanwise wave number $\beta$. This could lead to unrealistic computational time in a RANS code. In that way, some more practical methods involving a database approach or transition criteria have been developed and are introduced next.

\section{Database Method}

A database method is a simplified stability approach, which consists in storing or computing instability growth rates as a function of the mean-flow parameters (e.g., $[18,19])$.

One of these approaches, called parabola method [20], has been extensively developed at ONERA-The French Aerospace Lab for many years. It relies on the fact that, in a 2-D flow, for a given dimensionless frequency $F=2 \pi \nu f / U_{e}^{2}$, the evolution of the growth rate $\alpha_{i}$ as a function of the displacement-thickness Reynolds number $R e_{\delta 1}$ can be approximated by two half-parabolas. The coefficients of these parabolas only depend on the shape factor $H_{i}$ and the freestream Mach number $M_{e}$. This means that, if $M_{e}, \delta_{1}$, and $R e$ are known at a mesh point, the growth rate can easily and rapidly be calculated. The $N$ factor is then computed [Eq. (3)] and compared to the transition threshold $N_{T}$.

This method has been extended to 3-D flows [21], for which the CF amplification rate depends on the mean-velocity value and the shear stress at the inflection point of the transverse-velocity profile.

Thus, a database method appears as an interesting alternative to the linear-stability theory, because it provides the amplification rates easily and rapidly. In this study, this database method has been used in combination with a boundary-layer code. Such an approach involving boundary-layer computations with the database transition prediction has already provided interesting results for a laminar nacelle [22], and transonic [23] or supersonic laminar wings [24]. The use of database methods directly implemented in a RANS solver has been proposed by Bégou et al. [25] and has demonstrated promising results for 2 -D configurations. The main hurdle consisting in the integration of the growth rate [Eq. (3)] has been overcome with a transport equation for the $N$ factor.

\section{Transition Criteria}

Transition criteria represent a compromise between accuracy and ease of use. The fundamental mechanism of the transition process (i.e., amplification of boundary-layer modes) does not clearly appear as in the linear-stability analysis or database methods. The objective of transition criteria is to compare boundary-layer integral quantities to a threshold value: the beginning of transition starting where this threshold is reached.

In this study, the Arnal-Habiballah-Delcourt (AHD) criterion [26-28] (completed by the Gleyzes et al. [29] criterion in the case of a separation bubble) in its compressible formulation has been used in the RANS solver to calculate the TS-induced transition. The AHD criterion will detect transition if the Reynolds number $R e_{\theta}$ based on the boundary-layer momentum thickness overcomes a critical value. The latter depends on the upstream turbulence level Tu and the local pressure gradient (through the Pohlhausen parameter). More details about the AHD-Gleyzes (AHD-GL) criteria are available in the paper dealing with transition prediction and drag assessment for 2-D airfoils [10]. 
To compute the CF-induced transition in RANS computations, the so-called $C_{1}$ criterion has been used. It consists of an extension of the Beasley criterion [30], which predicts the CF-induced transition onset when the CF Reynolds number $R e_{\delta_{2}}$ defined in Eq. (7) is higher than $\left|R e_{\delta_{2}}\right| \geq 150$.

$$
R e_{\delta_{2}}=\frac{U_{e} \cdot \delta_{2}}{\nu_{e}} \quad \text { with } \delta_{2}=-\int_{0}^{\delta} \frac{\rho \cdot V}{\rho_{e} \cdot U_{e}} \mathrm{~d} z
$$

Using a number of wind-tunnel experiments on swept wings, Arnal et al. [26] incorporated the influence of the shape factor $H$ in the criterion leading to

$$
\operatorname{Re}_{\delta_{2}, T}= \begin{cases}150 & \text { if } H \leq 2.3 \\ \frac{300}{\pi} \arctan \left[\frac{0.106}{(H-2.3)^{2.05}}\right] & \text { if } 2.3<H \leq 2.7\end{cases}
$$

A limitation concerning the AHD-GL and $C_{1}$ criteria is that transition calculations are conducted along specific mesh lines (integration line concept), along which information has to be convected. These lines must be defined by the user before launching the RANS computation.

\section{E. Overset Grids, RANS Solver, and Far-Field Drag-Extraction Code}

The CFD computations were carried out with the wind-tunnel model dimensions, but run in free air, meaning that the support system and walls are not modeled. Moreover, only a halfconfiguration was considered, and the U-shape tail of the aircraft was not taken into account in the calculations because its role on the main wing transition is expected to be negligible.

Given the relative complexity of the LSBJ configuration, the overset approach was chosen to build an appropriate mesh for the structured RANS solver. These overset grids were generated following a methodology developed at ONERA-The French Aerospace Lab, which has been presented and validated in detail in a recent publication [31]. This strategy is based on the key concept of partitioning the computational domain: the near-body areas are meshed by a set of separate body-fitted grids generated with the grid generator Pointwise [32], whereas the off-body domain is treated with an automated Cartesian-grid method. This state-of-the-art combination allows a complex geometry to be considered as the sum of simple elements, such as fuselage, wing, nacelle, pylon, etc.

As an illustration, the wing near-body grid is shown in Fig. 3. It can be noted that it is a basic O-type grid; this is also the case for the fuselage, nacelle, and pylon grids. The wing chord is defined by 165 points, whereas the span is discretized by 221 points. There are 101 points in the normal direction: the boundary-layer area is described by a fine grid generated with a small stretch ratio of about 1.10 .
The wing grid counts 7.5 million cells: its refinement is largely satisfactory for RANS purposes. The normalized first cell height $Y^{+}$ on the wing has been verified a posteriori: it is between 0.25 and 1.3 for the Reynolds number considered in this study. Figure 4 shows a section of this grid at $35 \%$ of the aircraft span. The meshes of the nacelle, pylon, and fuselage have refinement levels that are consistent with the wing-grid characteristics.

Then, the off-body Cartesian mesh overlapping near-body grids is generated automatically using in-house software. The extent of this domain is about 150 chords in every direction from the aircraft (except toward the symmetry plane). A strong grid refinement is encountered in the vicinity of the LSBJ configuration: it leads to a size of 6 million cells for the Cartesian mesh.

As explained in [31], overset techniques imply blanking and overlapping management between the different structured grids. Indeed, cells of a given grid can be inside solid bodies of other grids. The masking step, which consists in removing such cells, as they have no physical sense, is necessary for the computation. The whole mesh used for the LSBJ configuration (without tail) is made of about 28 million elements, as shown in Fig. 5. As it can be noticed, the Cartesian mesh remains refined in a large zone surrounding the aircraft.

All the RANS computations of this study were performed with the solver elsA [33]. This software uses a cell-centered finite volume discretization on structured grids. Time integration is carried out by a backward Euler scheme with implicit relaxation. Spatial discretization is realized using a second-order-accuracy central Jameson et al. scheme [34] with artificial viscosity. Multigrid techniques were used to accelerate the convergence. Turbulence effects were simulated by the one-equation Spalart-Allmaras (SA) model [35].

At this stage, it should be mentioned that a large flow-separation region appeared at the upper nacelle-pylon junction in the first set of elsA computations. This flow separation, which is expected to have very limited effects on the wing-transition mechanisms, had negative impact on the numerical convergence. As a consequence, it was chosen to use only the SA-QCR2000 version [36] instead of the standard SA model. QCR stands for quadratic constitutive relation; this nonlinear version is not based on the traditional Boussinesq relation and is considered to produce more physical results in junction areas. The convergence was strongly improved. As shown in [37], for an aircraft configuration that does not exhibit massive flow separation on the wing at design point, the use of QCR with the SA model has very limited impact on performance prediction at cruise conditions.

To calculate transition in elsA, the combined AHD-GL criterion with a turbulence level of $0.1 \%$, which corresponds to a threshold transition factor close to 8 (see [10] for more details), and the $C_{1}$ criterion are used. Transition calculation is only applied to the wing while all the other aircraft elements are fully turbulent.

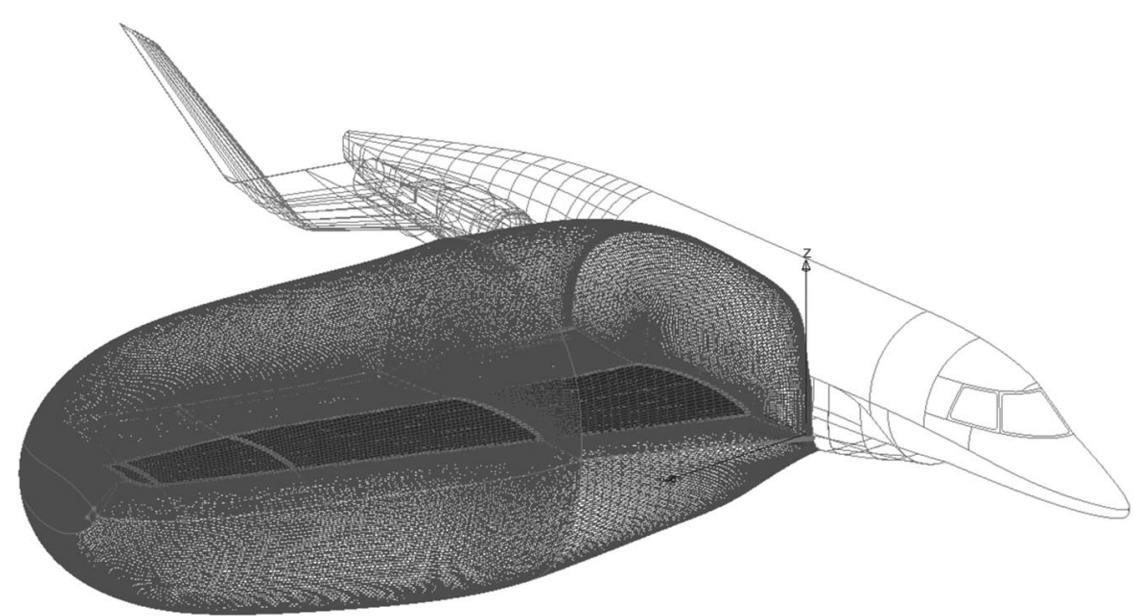

Fig. 3 Near-body mesh of the LSBJ wing. 


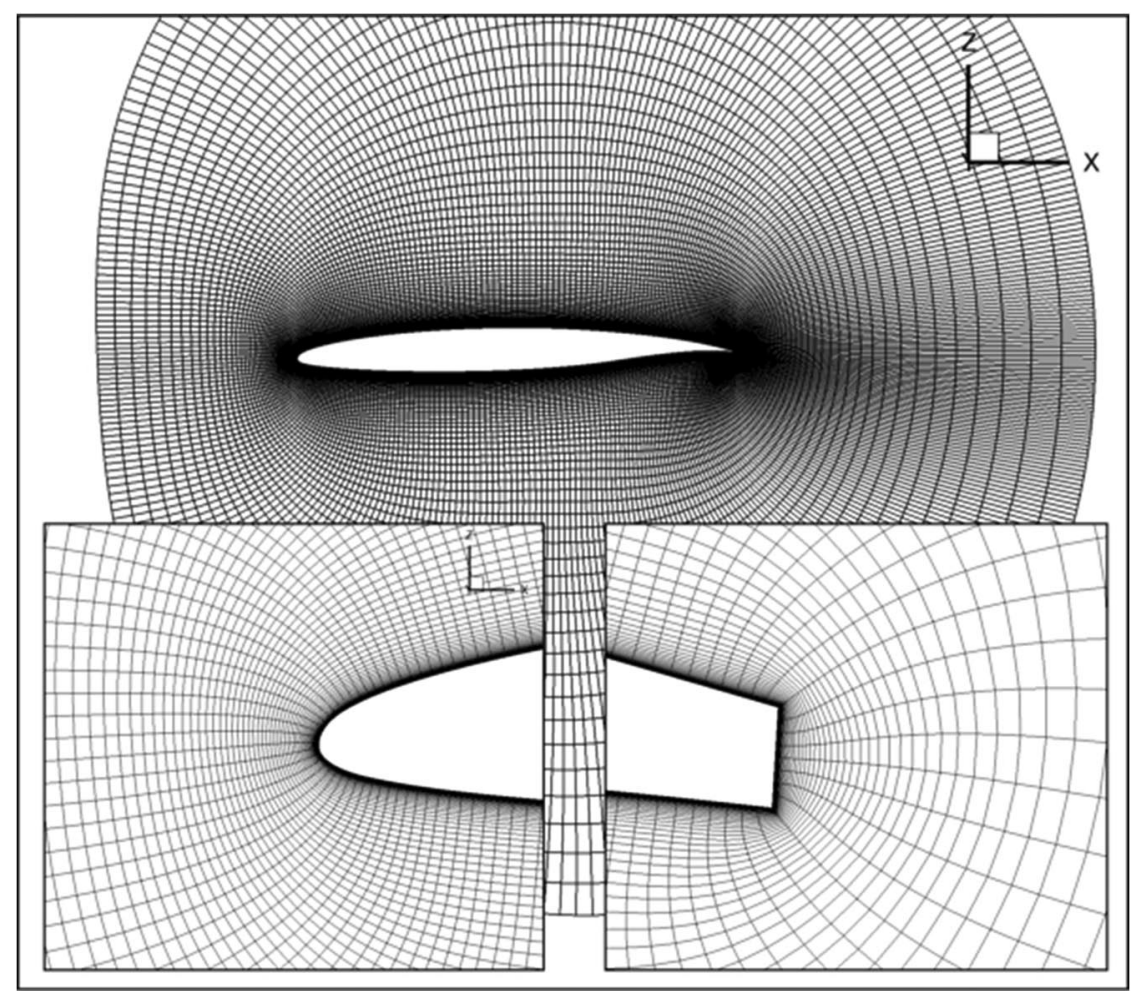

Fig. 4 Near-body mesh of the LSBJ wing at 35\% span.

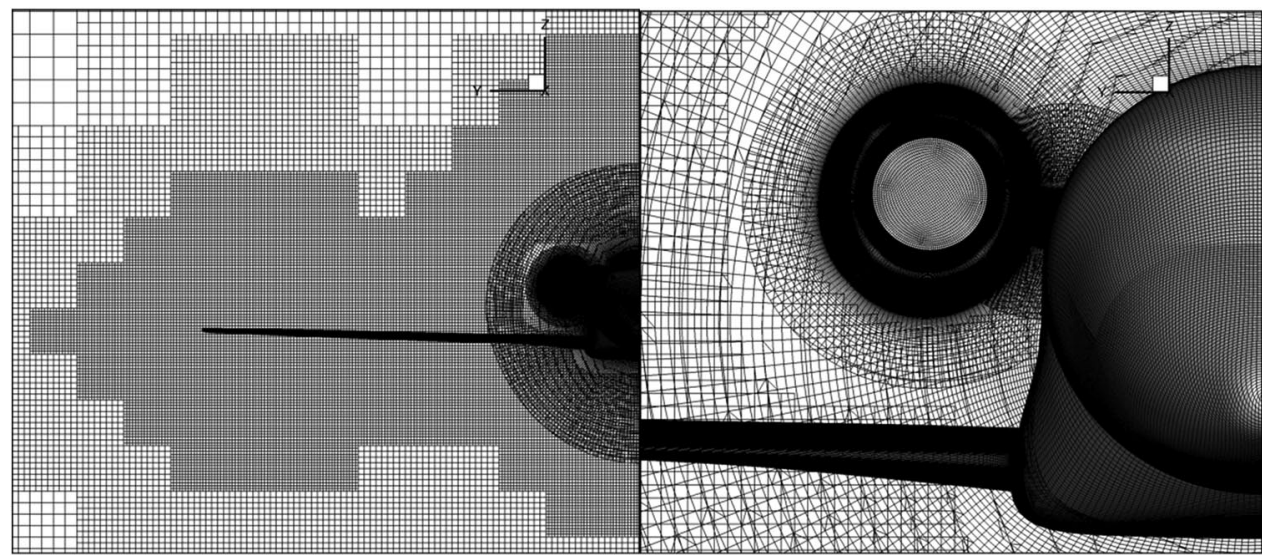

Fig. 5 Global view of the complete LSBJ mesh.

The calculations were carried out following the wind-tunnel operating conditions $\left(M a, P_{i}\right.$, and $\left.T_{i}\right)$. To reach a satisfactory level of convergence, the computations were continued until the fluxes were stable enough to observe a lift variation inferior to \pm 0.001 and a drag variation inferior to $1 \mathrm{drag}$ count $\left(10^{-4}\right)$ over the last thousand iterations. At least 4000 iterations using multigrid techniques (one coarse-grid level) were performed for all the calculations.

To compute the drag via CFD, two methods can be applied: the usual near-field approach uses skin integrations only, whereas the far-field approach consists in integrating values in the flow domain $[38,39]$. In this study, far-field analyses are carried out with the drag-extraction code ffd72 developed at ONERA-The French Aerospace Lab.

It can provide a physical drag breakdown into viscous $C_{D_{\nu}}$, wave $C_{D_{w}}$, and lift-induced $C_{D_{i}}$ components, and therefore eliminate the artificial or spurious drag $C_{D_{\mathrm{sp}}}$ by subtracting the near-field drag coefficient.

The different drag coefficients that are used in this paper are defined here:

$$
\begin{gathered}
C D_{\mathrm{nf}}=C D_{p}+C D_{f} \\
C D_{\mathrm{ff}}=C D_{v}+C D_{w}+C D_{i} \\
C D_{\mathrm{sp}}=C D_{\mathrm{nf}}-C D_{\mathrm{ff}}
\end{gathered}
$$

For the understanding of the following outcomes, the viscouspressure drag $C D_{\text {vp }}$ must be defined. It is the component of the viscous drag, which is not due to the friction, but to displacement effect, flow separation, etc.:

$$
C D_{\mathrm{vp}}=C D_{v}-C D_{f}
$$

The far-field formulation allows for the following near-field/far-field drag balance: 


$$
C D_{p}+C D_{f}=C D_{v}+C D_{w}+C D_{i}+C D_{\mathrm{sp}}
$$

To conclude on the numerical methods, transition analyses were first conducted using the RANS solver based on criteria (AHD-GL/ $C_{1}$ ). A database approach was then used in an in-house boundary-layer code. To conduct these boundary-layer computations, laminar RANS simulations (criteria inactivated) have been undertaken; the velocity field $\left(U_{e}, V_{e}, W_{e}\right)$ at the edge of the boundary layer was extracted and was used as an input for the boundary-layer code. This avoids using the infinite swept-wing assumption and allows a fully 3-D boundary-layer resolution. Additionally, in some specific regions, linear-stability calculations were performed to validate the database method.

\section{Transition-Prediction Results}

In this section, the numerical transition predictions will be evaluated and compared to cryoTSP visualizations. The considered cases correspond to a Mach number $M a=0.75$ for a Reynolds number $R e=9.75 \times 10^{6}$ based on the mean aerodynamic chord. Three different lift coefficients (or angles of attack) are presented; it allows three transition processes to be illustrated: pure TS-induced transition $\left(C_{L}=0.29\right)$, a pure CF-induced transition $\left(C_{L}=0.8\right)$, and a mixed transition scenario corresponding to the design point $\left(C_{L}=0.56\right)$ for which the TS and CF instabilities are significantly amplified. These analyses tend to focus on the wing pressure side because the transition on the suction side generally occurs at the shock position.

\section{A. TS-Induced Transition}

Firstly, a low angle of attack corresponding to $C_{L}=0.29$ has been considered. In this case, the transition to turbulence is driven by the amplification of TS instabilities on the wing pressure side. A comparison between the numerical $K_{p}$ distribution provided by elsA and the wall pressure-tap measurement is presented in Fig. 6 for three spanwise locations $y / s=34,46$, and $70 \%$. A target lift-coefficient procedure has been employed so that numerical lift coefficients match with the experimental ones. $C_{L}=0.29$ was reached for an experimental angle of attack of $0.67 \mathrm{deg}$ and a slightly lower value of $0.5 \mathrm{deg}$ numerically. The chordwise pressure evolution in Fig. 6 demonstrates a good agreement between the elsA computation and ETW measurements, which validates the iso- $C_{L}$ approach.

The transition locations obtained by the three distinct numerical methods as well as experimentally are all represented in Fig. 7. The background corresponds to cryoTSP visualizations with dark laminar areas and bright turbulent ones. The measurements exhibit a limited laminar area between $y / s=23 \%$ and $y / s=44 \%$. Out of this area, cryoTSP does not display other laminar zones. The features of this limited laminar area are particularly well captured by the boundarylayer computation represented by the dashed line and using the database transition-prediction method. The good behavior of the database method is confirmed by several linear-stability computations whose transition locations correspond to the squares in Fig. 7 For this configuration, the 2-D TS waves $(\Psi=0 \mathrm{deg})$ are responsible for the

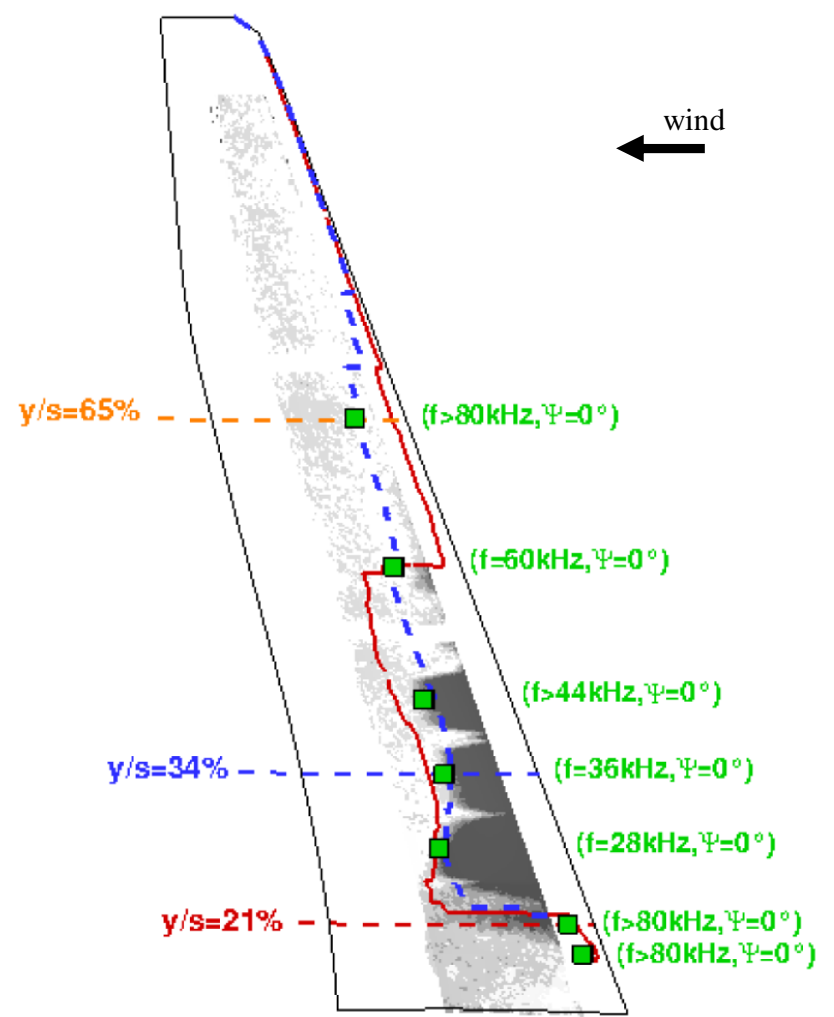

Fig. 7 Transition locations; $M a=0.75, C_{L}=0.29$; pressure side; background: cryoTSP visualization; full line: elsA; dashed line: database method; squares: TS-induced transition by linear-stability theory with most unstable frequencies and directions specified in parentheses.

transition onset. In the laminar region, their most unstable frequencies, specified in parentheses in Fig. 7, range from about 25 to $45 \mathrm{kHz}$. The transition location corresponding to the RANS computation with criteria corresponds to the full line. The AHD criterion captures the laminar area with only moderate deviation from the measurements even though its outboard extent is overestimated.

The comparison between the chordwise evolution of the TS $N$ factor (along $y / s=34 \%$ ) provided by the database approach (dashed line) and the exact stability computation (full line) is depicted in Fig. 8a. In this figure, the evolution of external velocity $U_{e}$ (thick line) and the stationary CF $N$ factor (dashed line) is also represented. It highlights the fact that, because the pressure gradient is weak from the close vicinity of the leading edge, the stationary $\mathrm{CF}$ instabilities are very weakly amplified with $N_{\mathrm{CF}_{0}}<2$. On the contrary, the TS $N$ factor reaches significant values beyond the transition threshold.

The existence of the limited laminar area between 23 and $44 \%$ of span can be explained by considering the chordwise pressure evolution plotted in Fig. 8b. Outside of the laminar area, along $y / s=21 \%$ and $y / s=65 \%$, the $K_{p}$ evolution shows a strong suction peak followed by

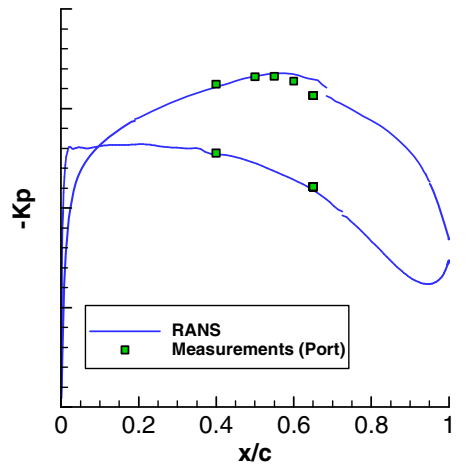

a) $\mathbf{y} / \mathbf{s}=\mathbf{3 4 \%}$

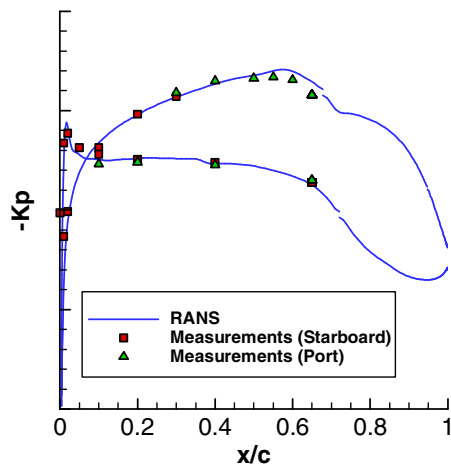

b) $\mathrm{y} / \mathrm{s}=\mathbf{4 6 \%}$

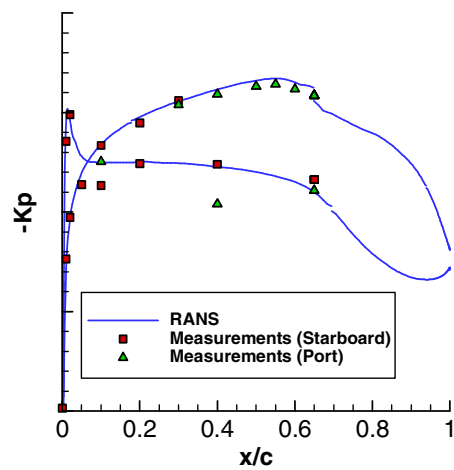

c) $\mathbf{y} / \mathrm{s}=\mathbf{7 0} \%$

Fig. 6 Pressure distribution; $M a=0.75, C_{L}=0.29$; comparison between measurements (symbols) and elsA (lines). 


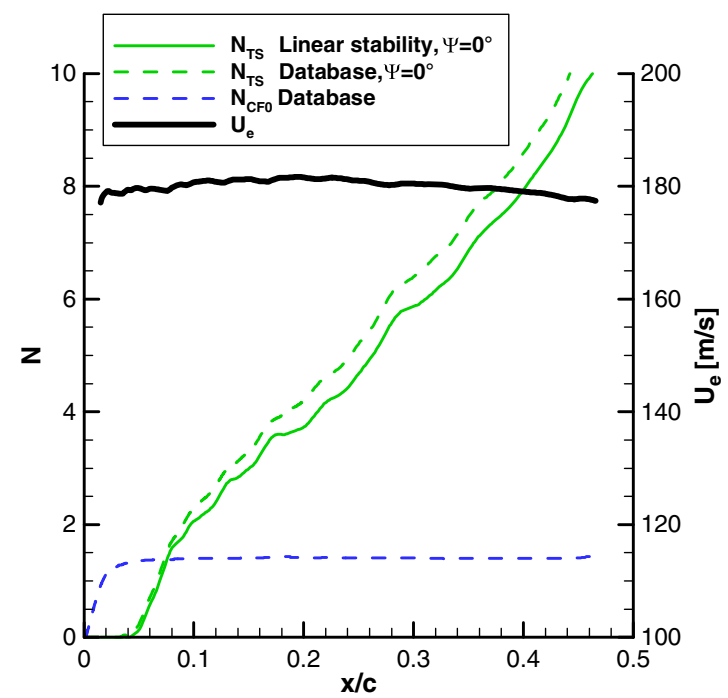

a) $N$ factors chordwise evolution along $y / s=34 \%$; dashed lines: database, full line: linear stability theory; TS instability, CF instability, thick black: external velocity distribution

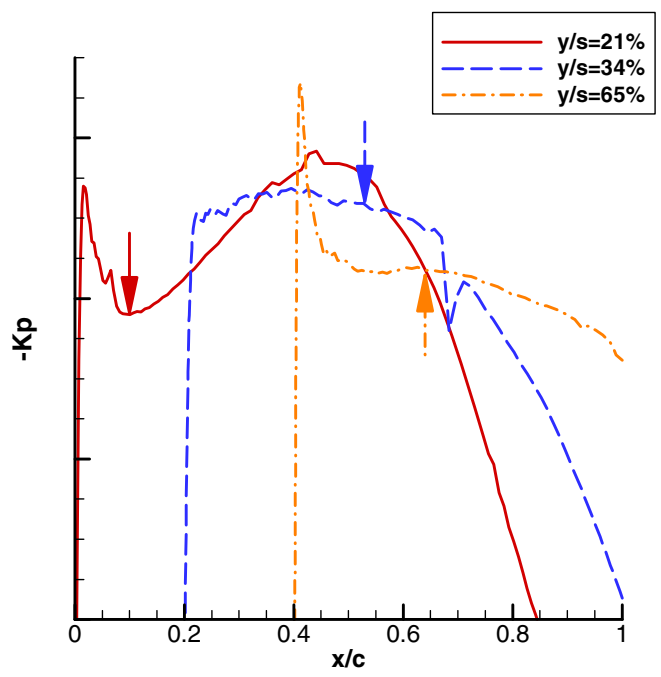

b) Chordwise evolution of pressure coefficient along three spanwise stations; the vertical arrows: TS induced transition location provided by database approach; for sake of better readability, the plots have been offset by 0.2

Fig. $8 \mathrm{~N}$-factor and pressure-coefficient chordwise evolutions; $M a=0.75, C_{L}=0.29$, pressure side.

a steep deceleration, which induces a rapid TS transition, as depicted by the vertical arrows. In this strongly decelerated region, the TS modes are characterized by very high frequencies above $f>80 \mathrm{kHz}$. Inside the laminar area, at $y / s=34 \%$, the suction peak is significantly reduced so that a TS-induced transition occurs further downstream.

\section{B. CF-Induced Transition}

A transition triggered by the $\mathrm{CF}$ amplification is observed on the pressure side of the wing for a lift coefficient of 0.8 corresponding to an angle of attack of $3.9 \mathrm{deg}$ with elsA (4.2 deg in the tests). Transition locations are plotted in Fig. 9. The legend is the same as for Fig. 7 except that the squares corresponding to the TS-induced transition

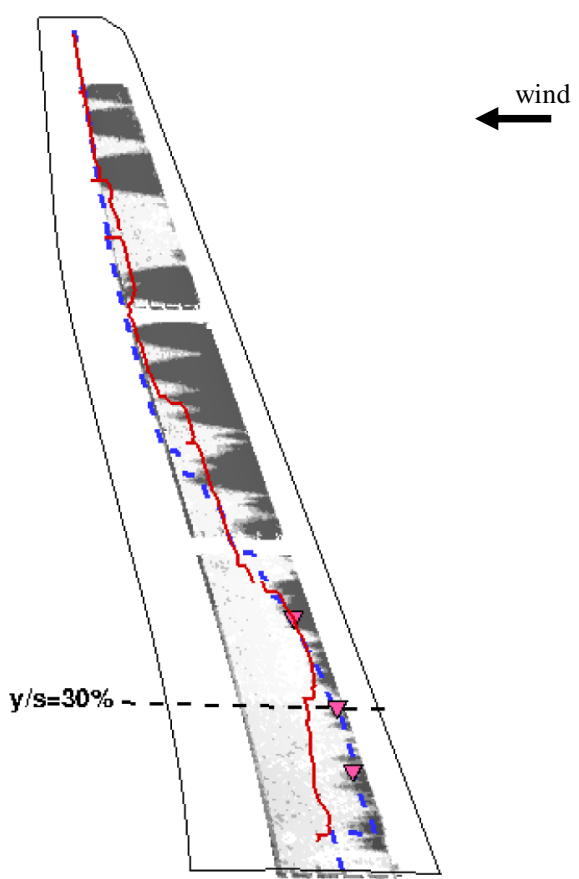

Fig. 9 Transition locations; $M a=0.75, C_{L}=0.8$; legend: see Fig. 7; downward-pointing triangles: stationary $\mathrm{CF}$-induced transition provided by linear-stability theory. have been replaced by downward-pointing triangles standing for the $\mathrm{CF}$-induced transition provided by the linear-stability theory.

In the inboard part of the wing (i.e., up to midspan), the TSP visualization shows a CF-induced transition. This is well captured by the boundary-layer computation with the database method (dashed line), which is once again validated by the linear-stability theory, as depicted by the downward-pointing triangles. The transitionprediction line from the RANS computation (full line in Fig. 9) demonstrates that the $C_{1}$ criterion provides quite satisfactory results even though it predicts a transition onset about $10 \%$ downstream of measurements between the root and $y / s=30 \%$.

In the outboard part of the wing (i.e., $y / s>50 \%$ ), stability analyses (both the database and the linear-stability theory) show that the amplification of disturbances is moderate with $N$ factors that remain below the transition threshold. This demonstrates that the boundary layer remains laminar up to the minimum pressure point, meaning that a laminar separation occurs.

A comparison between the stationary $\mathrm{CF} N$ factor approximated by the database method (dashed line) and by the linear-stability theory (full line) is illustrated in Fig. 10. The agreement is fairly good and provides a close transition location at the critical value $N_{T, \mathrm{CF}_{0}}=8$. The chordwise evolution of the external velocity $U_{e}$, depicted by the thick full line, shows that the flow is continuously accelerated, which leads to a strong amplification of CF instabilities: $N_{\mathrm{CF}_{0}}>10$ at midchord. At the same time, the TS instabilities $(\Psi=40 \mathrm{deg})$ are slightly amplified with $N_{\mathrm{TS}}<5$.

The evolution of the Reynolds number based on the transverse boundary-layer thickness $R_{\delta 2}$ is represented in Fig. 11a. It shows that, in the inboard region, $R_{\delta 2}$ is high and exceeds its critical value around 150 corresponding to the $C_{1}$ criterion threshold [Eq. (8)]. Furthermore, the chordwise evolution of the pressure coefficient (Fig. 11b) clearly shows that, in the inboard region, the flow is strongly accelerated leading to a CF-induced transition upstream of the minimum pressure point. This is not the case anymore at $y / s=67 \%$ where transition occurs due to separation.

\section{Cruise Conditions: Mixed Transition Process}

The same analysis has been applied to a case close to the design point: $M a=0.75, C_{L}=0.56, R e=9.75 \times 10^{6}$. Transition locations are represented in Fig. 12 for the suction side (Fig. 12a) and the pressure side (Fig. 12b). On the suction side, the database method and the linearstability theory provide moderate $N$ factors, which remain below the 


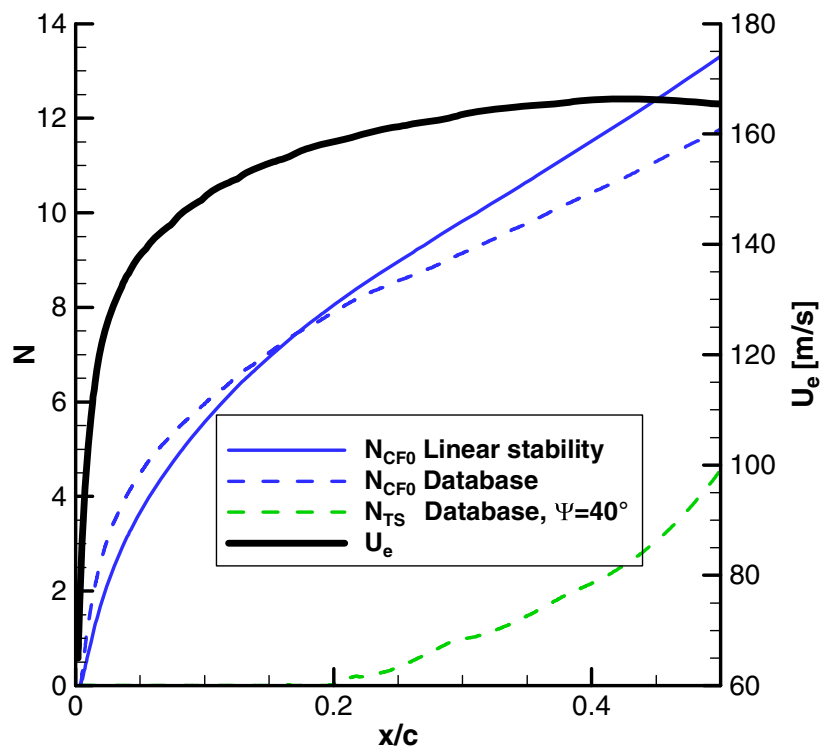

Fig. $10 N$-factor chordwise evolutions along $y / s=30 \% ; M a=0.75$, $C_{L}=0.8$.

transitional critical value. In the same way, the RANS simulation shows that neither the AHD criterion nor the $C_{1}$ criterion is triggered. This demonstrates that the boundary layer remains laminar up to the shock located at around $60 \%$ of chord.

On the pressure side, the transition process is more complicated. As in the previous case, beyond midspan, the boundary layer remains laminar up to the minimum pressure point, meaning that the transition is induced by boundary-layer separation. This is again well captured by numerical methods (RANS criteria or database method), and confirmed by an exact stability computation (upward-pointing triangle in Fig. 12b) at $y / s=84 \%$, which provides low $N$ factors: $N_{\mathrm{TS}}<6$ and $N_{\mathrm{CF}, 0}<1$.

In the inboard wing, there is a strong competition between the TS and $\mathrm{CF}$ instabilities. This is illustrated in Fig. 13, at $y / s=30 \%$ (Fig. 13a) and $y / s=34 \%$ (Fig. 13b), which compares the chordwise evolution of $N$ factors provided by the linear-stability theory (full lines) and the database method (dashed lines) for oblique TS instabilities and stationary crossflow vortices. To analyze the influence of the pressure gradient on modal instabilities, the evolution of the freestream velocity

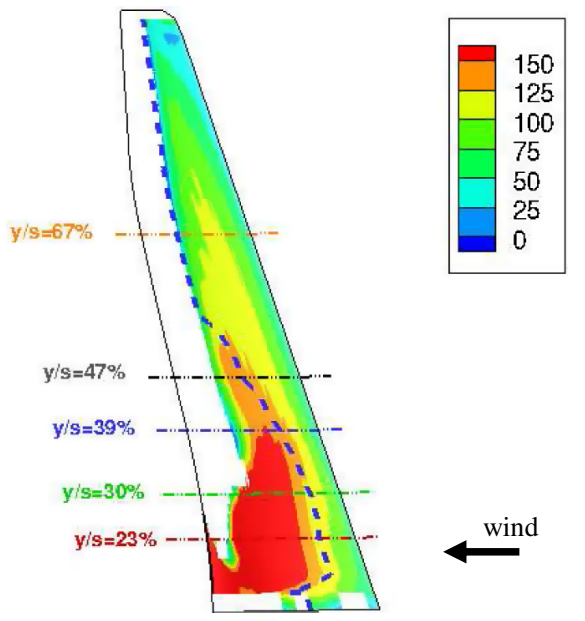

a) Background: $R_{\delta 2}$ values on the whole part of the wing; dashed line: transition line given by database method is still represented by the thick line. The TS waves begin to be amplified around $x / c \approx 15 \%$ where the external velocity gradient in the streamwise direction becomes moderate. Upstream, the strong acceleration stabilizes the TS instabilities. The linear-stability theory provides unstable frequencies around $25 \mathrm{kHz}$ for this configuration; the most unstable direction is $\Psi=40 \mathrm{deg}$. The agreement concerning the $N$ factors for oblique TS waves between the linear-stability theory and the database method is very good. The stationary crossflow vortices are strongly amplified from the separation line up to $x / c \approx 10 \%$. Downstream, as the flow remains fairly accelerated, $\mathrm{CF}$ waves are still amplified. The database approach provides results relatively close to the linear-stability theory: nonetheless, the $\mathrm{CF}$ growth rate is slightly overestimated up to $10 \%$ of chord, whereas it is underestimated downstream. As a result, whereas the $\mathrm{CF} N$ factor from the linear-stability theory exceeds the transition value, the CF $N$ factor from the database remains lower than $N_{T, \mathrm{CF}}=8$ before TS onset. This means that the linear-stability theory predicts a CF-induced transition contrary to the database method. The database method for $\mathrm{CF}$ instabilities is based on the mean-flow properties at the general inflection point of the velocity profile in the wall-normal direction. Even though the chordwise $N$ factor evolution is rather well represented by the database approach, this case shows that the modeling could be improved.

In conclusion for the inboard wing, the linear-stability theory provides a CF-induced transition (downward-pointing triangles in Fig. 12b) slightly upstream of the TS transition (squares). Because of the discrepancies concerning the CF $N$ factors, the database method would instead predict a TS-induced transition (dashed line). Finally, in this part of the wing, the RANS computation (full line in Fig. 12b) provides a transition line $\left(C_{1}\right)$ slightly closer to the leading $\overline{\text { edge }}$ compared to the other numerical data and cryoTSP visualization. From a qualitative point of view and despite the visible differences, the shape of the transition line is well captured by elsA.

In this section, the ability of numerical tools to predict transition onset has been compared to TSP measurements. The database approach provides very accurate results in close agreement with the experiments. Nonetheless, at this time, this method has not been integrated into a RANS solver. Even though discrepancies due to the $C_{1}$ criterion behavior exist for the RANS transition prediction, the results via this approach are globally satisfactory.

\section{Performance Prediction and Gains}

This section highlights the performance benefits due to the extended laminar flow that is obtained over the wing. The gains are

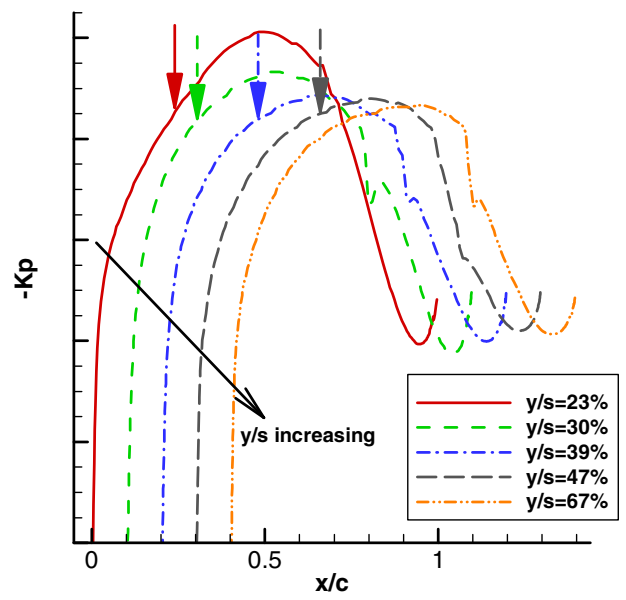

b) Chordwise evolution of pressure coefficient along five spanwise stations; the vertical arrows: $\mathrm{CF}$-induced transition location provided by database approach; for sake of better readability, the plots have been offset by 0.1

Fig. 11 Transverse-thickness Reynolds number and pressure-coefficient evolution; $M a=0.75, C_{L}=0.8$; pressure side. 


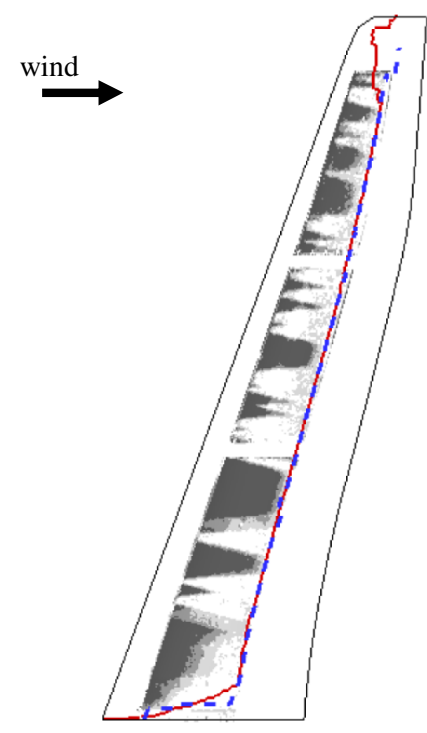

a) Suction side

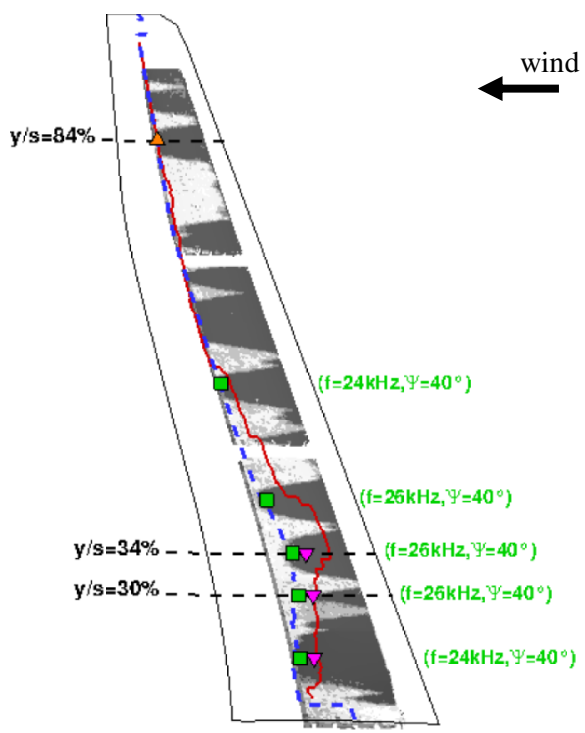

b) Pressure side

Fig. 12 Transition locations; $M a=0.75, C_{L}=\mathbf{0 . 5 6}$; legend: see Fig. $\underline{7}$; upward-pointing triangle: separation.

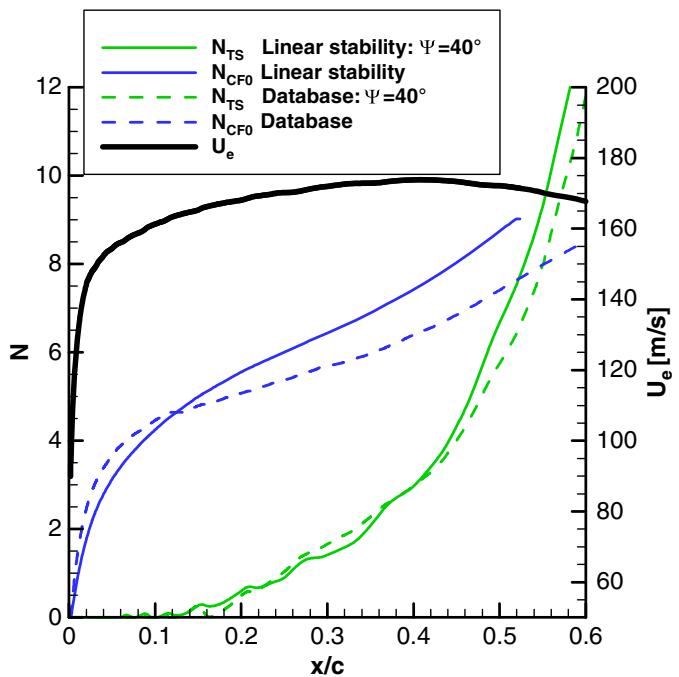

a) $\mathbf{y} / \mathbf{s}=\mathbf{3 0 \%}$

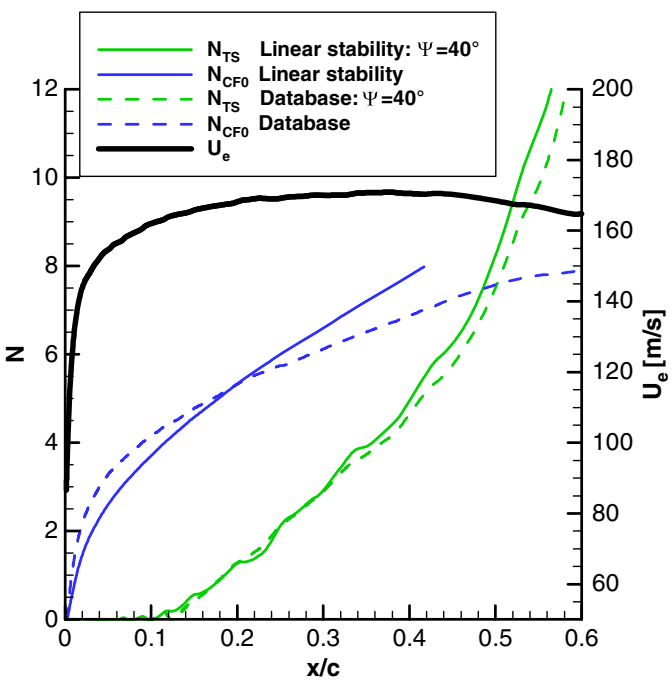

b) $\mathbf{y} / \mathbf{s}=\mathbf{3 4 \%}$

Fig. $13 N$-factor chordwise evolutions; design point $M a=0.75, R e_{c}=9.75 \times 10^{6}, C_{L}=0.56$; legend: see Fig. $\underline{\text { a }}$.

evaluated by comparing natural laminar cases with turbulent ones both via numerical and experimental approaches. For the laminar cases, numerical data are obtained using elsA with the AHD-GL and $C_{1}$ criteria $\left(T u=0.1 \% / N_{T}=8\right)$.

It is important to keep in mind that some differences exist between the elsA computations and the ETW tests that have been carried out. Several of these differences only affect the absolute comparisons between numerical and experimental data, such as free air vs wind-tunnel section with uncorrected support-system effects; fully turbulent fuselage, pylon, and nacelle in CFD while transition was not triggered on these components for both laminar and turbulent wind-tunnel runs; limitations of the RANS models for the description of the massive flow separation at the nacelle-pylon junction, etc. Other differences may impact relative comparisons, and therefore the assessment of performance benefits: first, as seen in the previous section, the laminar flow over the ETW wing model exhibited many turbulent wedges, and then the turbulent cases were fully turbulent in CFD, whereas the transition was only triggered between 5 and $15 \%$ of the wing chord for the turbulent runs of the test campaign.
Beyond these elements, the numerical and experimental cases that have been chosen for this section exhibit a satisfactory global agreement in terms of wing-transition location.

As the Mach number has a significant effect both on laminar-flow extent and global aircraft performance, the results obtained with two different values will be presented. Consequently, the conditions will be the following: $M a=0.75, P_{i}=1.38$ bars, $T_{i}=120 \mathrm{~K} ;$ and $M a=0.78, P_{i}=1.35$ bars, $T_{i}=120 \mathrm{~K}$. They both correspond to a Reynolds number based on the mean chord of about 10 million. A large range of lift coefficients has been investigated: $0.20<C_{L}<1.00$.

For confidentiality reasons, in this section dedicated to the sensitive topic of performance, the scales of the figures showing absolute comparisons are blanked.

Concerning the aircraft lift coefficient, the gain due to laminar flow over the wing is highlighted in Fig. 14. Both numerical and experimental values are given. It can be noticed that, even if discrepancies exist between the two approaches, the gains that they exhibit are quite similar. Both for measurements and computations, this difference between laminar and turbulent flows is almost constant over the whole polar with 


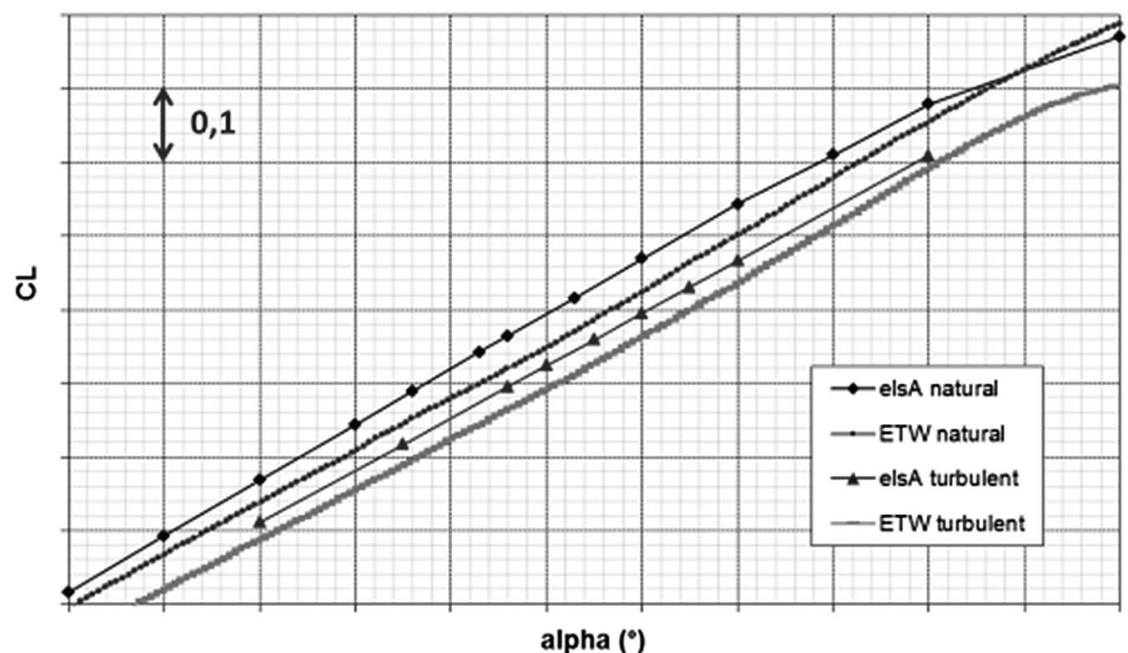

Fig. $14 C_{L}(\alpha)$; laminar vs turbulent (triggered) flows; $M a=0.75$.

a slight increase for the highest angles of attack. The gain in lift coefficient varies between 0.06 and 0.08 ; this is consistent with what was obtained with the 2-D configuration in [10]. It can be observed that the delta shown by elsA is slightly larger than the one from the experiments. This was expected because the experimental laminar cases encounter turbulent wedges and also because, in the turbulent wind-tunnel cases, $5-15 \%$ of the wing chord remains potentially laminar.

Concerning the aircraft near-field drag coefficient, the equivalent comparison is shown in Fig. 15. The drag reduction is visible over the whole range of lift coefficients. The experimental and numerical trends are very similar. Nevertheless, the CFD drag values are slightly lower than the experimental ones both for laminar and turbulent polars (only by a few drag counts). As mentioned previously, this could come from uncorrected support-system effects or from a significant difference in the prediction of the extended nacelle-pylon flow separation. The discrepancy between the CFD and wind-tunnel drags is larger for the laminar cases. The most probable reason is clearly the turbulent wedges that appeared on the model.

To analyze the drag reduction, Fig. 16 gives the experimental and numerical gains. The difference between the CFD and wind-tunnel gains varies between 3 and 6 drag counts, which is very limited considering the fact that, in the tests, the laminar zone was contaminated by numerous turbulent wedges. As expected, the gain obtained by elsA is greater. Nevertheless, the two curves are in satisfactory agreement and exhibit similar behaviors. The gain in drag strongly increases with the lift level: it doubles between $C_{L}=0.2$ and $C_{L}=0.8$. For lift coefficients corresponding to the cruise conditions, the drag reduction achieves several dozens of counts. It represents a saving of $10-15 \%$ of the aircraft drag.

To investigate the Mach-number effect on the drag-component productions, Fig. 17 presents the results from the far-field dragextraction code ffd $\overline{72}$ obtained with laminar computations at Machnumber values of 0.75 and 0.78 . The evolutions of friction, viscous pressure, and wave-drag values are highlighted. First, the beneficial impact of the Mach number on laminar flow can be observed here on the $C D_{f}$ coefficient. Indeed, the friction-drag level at $M a=0.78$ is lower by as much as six counts compared to the one at $M a=0.75$. Then, it can be noticed that it is not only the wave drag that causes drag rise for high $C_{L}$ coefficients at $M a=0.78$, but also the viscous-pressure component. The latter remains greater than the wave drag, and achieves values as large as those of the friction drag in this $C_{L}$ range. These observations are in good agreement with the ones obtained for the 2-D configuration [10].

To analyze the role of the viscous-pressure component in the drag reduction due to laminar regions at a given Mach number, Fig. 18 shows the numerical $C D_{f}$ and $C D_{\mathrm{vp}}$ coefficients both for the laminar and turbulent cases at $M a=0.75$. The wave-drag value (shockinduced drag) is not shown because it remains almost unchanged.

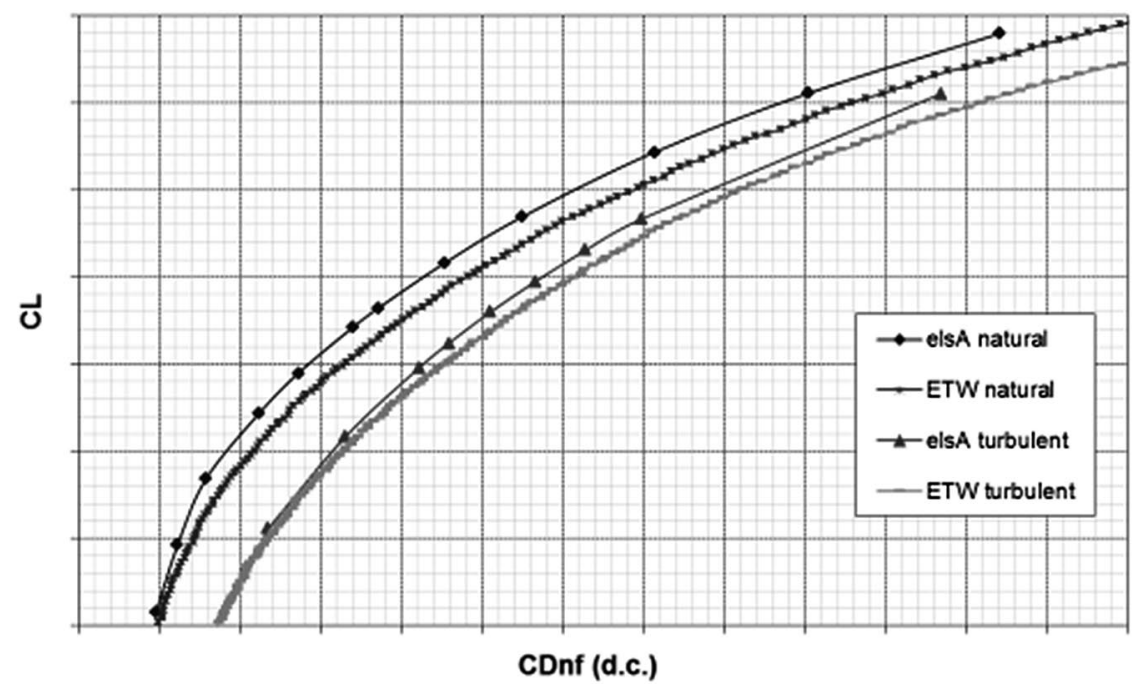

Fig. $15 C_{L}\left(C D_{\mathrm{nf}}\right)$; laminar vs turbulent (triggered) flows; $M a=0.75$. 


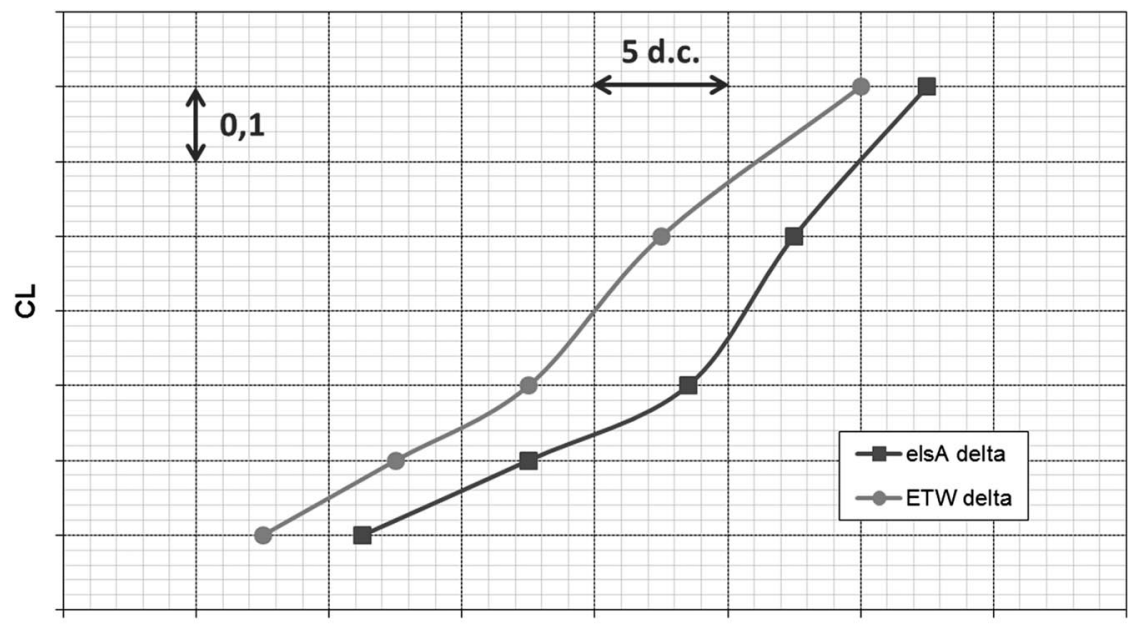

$\Delta \mathrm{CDnf}$ (d.c.)

Fig. $16 C_{L}\left(\Delta C D_{\mathrm{nf}}\right)$; numerical and experimental gains; $M a=0.75$.

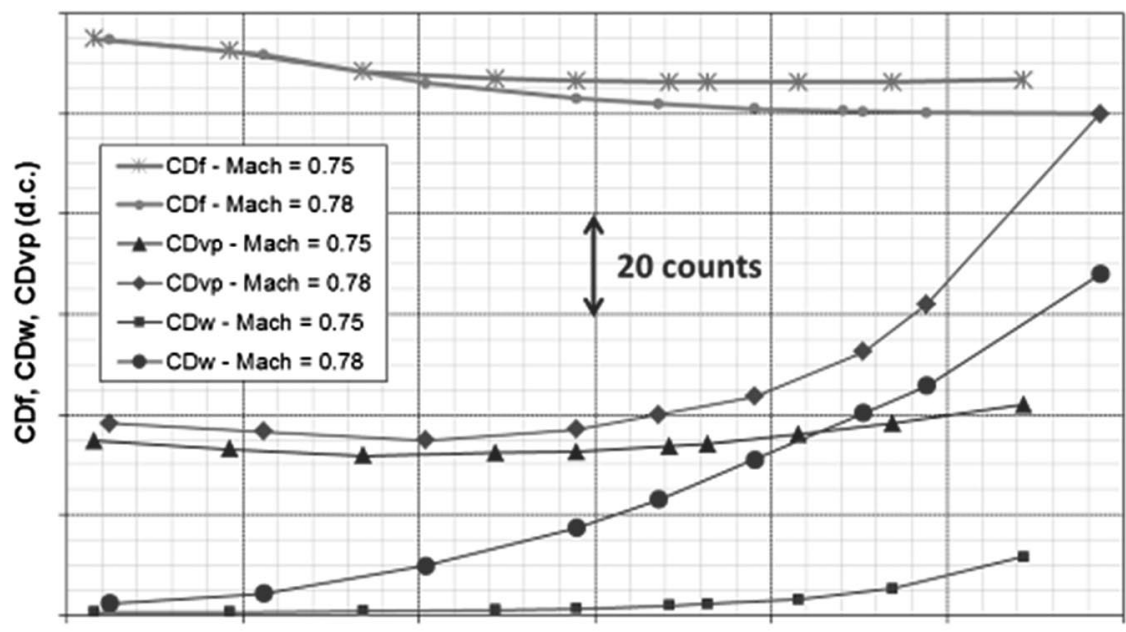

$\mathrm{CL}$

Fig. 17 Numerical far-field drag components; laminar flow; Mach-number effects.

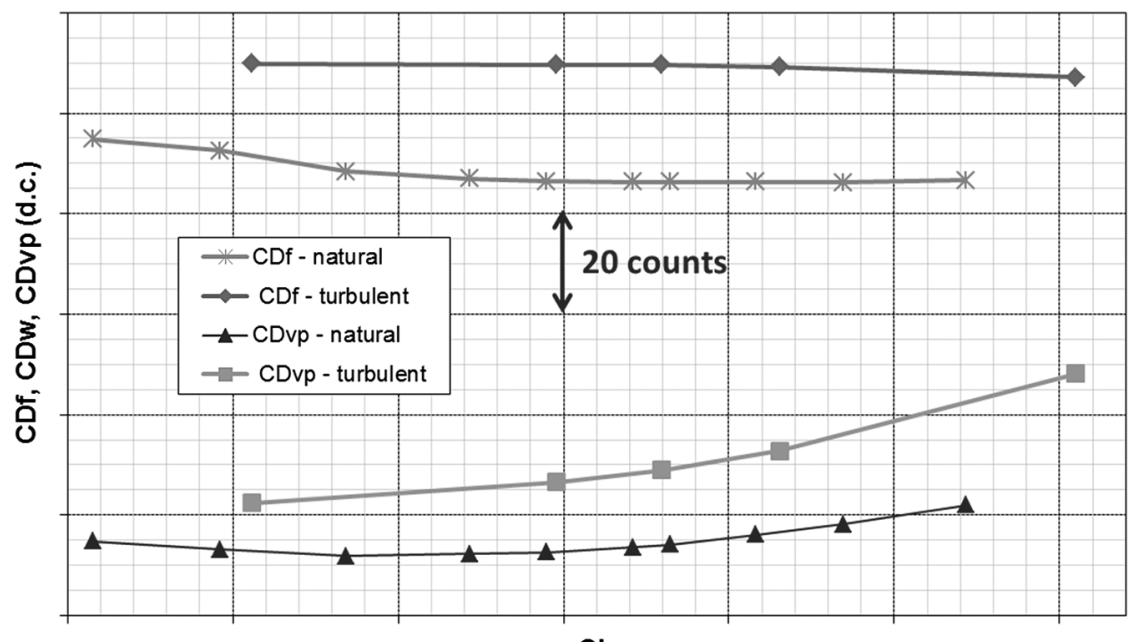

CL

Fig. 18 Numerical far-field drag components; laminar vs turbulent (triggered) flows; $M a=0.75$.

As with the 2-D laminar airfoil studied in [10], the resulting drag gain is a combination of friction and viscous-pressure coefficient decreases. The 2-D results basically exhibited a 50/50 contribution with an increasing role of $C D_{\mathrm{vp}}$ at high lift levels. This is in agreement with Fig. 18, but the contribution of the friction component seems larger for the aircraft configuration, especially at intermediate lift values. 


\section{Conclusions}

This paper focused on laminar-to-turbulent transition and performance predictions of a realistic business jet configuration fitted with laminar wings. It is the extension of the paper [10], which was limited to 2-D transition mechanisms. In the present study, highlevel experimental tests and numerical analyses have been carried out to further investigate the potential of this technology.

A test campaign was held at the end of 2015 in the European Transonic Windtunnel: it included force and moment measurements (both for laminar and triggered transition cases), dynamic and static pressure probing, as well as cryoTSP visualizations allowing the wing-transition locations to be determined. The flow conditions presented in this paper correspond to Mach numbers of 0.75 and 0.78 , and a chord Reynolds number close to 10 million. A large range of lift coefficients was investigated.

Numerous Reynolds-averaged Navier-Stokes (RANS) and boundary-layer computations, based on wind-tunnel test conditions, were performed. Three-dimensional transition mechanisms were computed by different numerical approaches. First, the Arnal-HabiballahDelcourt and Gleyzes et al. [29] transition criteria in combination with the $C_{1}$ crossflow criterion implemented in the elsA solver were used. Secondly, boundary-layer computations involving the databaseapproach transition prediction were performed. The velocity field at the edge of the boundary layer of fully laminar RANS simulations (with inactivated criteria) was used as an input for the boundary-layer code. This approach avoided the use of the infinite swept-wing assumption and allowed a fully 3-D boundary-layer resolution. Additionally, in some specific regions of interest, linear-stability calculations were carried out to validate the database method. The numerical results were then compared to the cryoTSP measurements.

The database approach provided results in close agreement with the test measurements. However, this method is currently not integrated into any RANS solver. As a consequence, the approach of RANS simulations with transition criteria is a good compromise that leads to results that are globally satisfactory and close to experimental values even if small discrepancies due to the $C_{1}$ criterion behavior were observed.

Considering the gains achieved with the laminar wing design, the numerical far-field approach was particularly useful to compare natural and triggered transition calculations. The role of the viscous-pressure component in drag reduction was highlighted once again. In cruise conditions, the total drag for this aircraft configuration was reduced by $10-15 \%$ due to the extended laminar flow over the wings, confirmed by numerical and experimental data.

\section{Acknowledgments}

The authors would like to thank the European Transonic Windtunnel (ETW) and DLR, German Aerospace Center teams for the efficient preparation and execution of the tests. This work has been undertaken within the Joint Technology Initiative Clean Sky, Smart Fixed Wing Aircraft Integrated Technology Demonstrator, a project financed by the Seventh Framework Programme of the European Commission under the following grant agreements: CSJU-GAM-SFWA-2008-001 (Dassault Aviation and ONERAThe French Aerospace Lab activities), IDAMME2 number 323362 (model design and manufacturing), and High-Speed Wind-Tunnel Test of a Laminar-Configuration Bizjet number 338517 (ETW test campaign).

\section{References}

[1] Joslin, R. D., "Overview of the Laminar Flow Control," NASA TP1998-208705, 1998, http://ntrs.nasa.gov/archive/nasa/casi.ntrs.nasa. gov/19980232017.pdf [retrieved Jan. 2017].

[2] Somers, D. M., "Subsonic Natural-Laminar Flow Airfoils," Natural Laminar Flow and Laminar Flow Control, edited by R. W. Barnwell, and M. Y. Hussaini, Springer-Verlag, New York, 1992, pp. $143-176$.

doi:10.1007/978-1-4612-2872-1_4
[3] Amoignon, O., Pralits, J., Hanifi, A., Berggen, M., and Henningson, D., "Shape Optimization for Delay of Laminar-Turbulent Transition," AIAA Journal, Vol. 44, No. 5, May 2006, pp. 1009-1024. doi:10.2514/1.12431

[4] Viken, J. K., Viken, S. A., Pfenninger, W., Morgan, H. L., Jr., and Campbell, R. L., "Design of the Low Speed NLF (1)-0414F and the High-Speed HSNLF(1)-0213 Airfoils with High Lift Systems," Research in Natural Laminar Flow and Laminar Flow Control, 1987, pp. 637-671; also NASA CP-2487, 1987, http://ntrs.nasa.gov/archive/ nasa/casi.ntrs.nasa.gov/19900003224.pdf [retrieved Jan. 2017].

[5] Sewall, W. G., McGhee, R. J., Hahne, D. E., and Jordan, F. L., Jr., "Wind Tunnel Results of the High-Speed NLF(1)-0213 Airfoil," Research in Natural Laminar Flow and Laminar Flow Control, Pt. 3, 1987, pp. 697726; also NASA CP-2487, 1987, http://ntrs.nasa.gov/archive/nasa/casi.ntrs. nasa.gov/19900003226.pdf [retrieved Jan. 2017].

[6] Sewall, W. G., McGhee, R. J., Viken, J. K., Waggoner, E. G., Walker, B. S., and Millard, B. F., "Wind Tunnel Results for a High-Speed, Natural Laminar-Flow Airfoil Designed for General Aviation Aircraft," NASA TM 87602, 1985, http://ntrs.nasa.gov/archive/nasa/casi.ntrs.nasa. gov/19880004696.pdf [retrieved Jan. 2017].

[7] Fujino, M., Yoshizaki, Y., and Kawamura, Y., "Natural-Laminar-Flow Airfoil Development for a Lightweight Business Jet," Journal of Aircraft, Vol. 40, No. 4, July-Aug. 2003, pp. 609-615. doi: $10.2514 / 2.3145$

[8] Perraud, J., Salah El Din, I., Schrauf, G., Hanifi, A., Donelli, R., Hein, S., Fey, U., Egami, Y., and Streit, T., "High Reynolds Number Transition Experiments in the ETW Test Facility with the Pathfinder Model," Proceedings of the 5th European Conference on Computational Fluid Dynamics, ECCOMAS CFD 2010, 2010.

[9] Streit, T., Horstmann, K. H., Schrauf, G., Hein, S., Fey, U., Egami, Y., Perraud, J., Salah El Din, I., Cella, U., and Quest, J., "Complementary Numerical and Experimental Data Analysis of the ETW Telfona Pathfinder Wing Transition Tests," 49th Aerospace Sciences Meeting, AIAA Paper 2011-881, Jan. 2011.

doi:10.2514/6.2011-881

[10] Hue, D., Vermeersch, O., Bailly, D., Brunet, V., and Forte, M., "Experimental and Numerical Methods for Transition and Drag Predictions of Laminar Airfoils," AIAA Journal, Vol. 53, No. 9, Sept. 2015, pp. 2694-2712. doi:10.2514/1.J053788

[11] Dougherty, N. S., Jr., and Fisher, D. F., "Boundary Layer Transition on a 10-Degree Cone: Wind Tunnel/Flight Data Correlation," 18th Aerospace Sciences Meeting, AIAA Paper 1980-0154, Jan. 1980. doi:10.2514/6.1980-154

[12] Quix, H., and Quest, J., "Hotwires in Pressurized, Cryogenic Environment-It Works!," 50th AIAA Aerospace Sciences Meeting, AIAA Paper 2012-0105, Jan. 2012. doi:10.2514/6.2012-105

[13] Van Ingen, J. L., "A Suggested Semi-Empirical Method for the Calculation of the Boundary-Layer Transition Region," Dept. of Aerospace Engineering, Univ. of Delft, Rept. VTH-74, Delft, The Netherlands, 1956.

[14] Smith, A. M. O., and Gamberoni, N., "Transition Pressure Gradient and Stability Theory," Douglas Aircraft, Rept. ES-26388, 1956.

[15] Mack, L. M., "Transition Prediction and Linear Stability Theory," AGARD Conference Proceedings, No. 224, Paris, 1977.

[16] Fey, U., Egami, Y., and Klein, C., "Using cryoTSP as a Tool for Transition Detection and Instability Examination at High Reynolds Numbers," New Results in Numerical and Experimental Fluid Mechanics VI, edited by C. Tropea, J. Suad, H.-J. Heinemann, R. Henke, and H. Hönlinger, Vol. 96, Springer-Verlag, Berlin, 2008, pp. $227-234$.

doi:10.1007/978-3-540-74460-3_28

[17] Klein, C., Henne, U., Costantini, M., Ondrus, V., and Beifuss, U., "Development of a Highly Sensitive Temperature-Sensitive Paint for Measurements Under Cryogenic Temperatures (100-160 K) Conditions," 54th AIAA Aerospace Sciences Meeting, AIAA Paper 2016-0650, Jan. 2016. doi: $10.2514 / 6.2016-0650$

[18] Van Ingen, J. L., "The $e^{N}$ Method for Transition Prediction: Historical Review of Work at TU Delft," 38th Fluid Dynamics Conference and Exhibit, AIAA Paper 2008-3830, June 2008. doi:10.2514/6.2008-3830.

[19] Drela, M., "Implicit Implementation of the Full $e^{N}$ Transition Criterion," 21st Applied Aerodynamics Conference, AIAA Paper 2003-4066, June 2003. doi: $10.2514 / 6.2003-4066$

[20] Arnal, D., "Transition Prediction in Transonic Flow," Symposium Transsonicum III, edited by J. Zierep, and H. Oertel, International 
Union of Theoretical and Applied Mechanics, Springer, Berlin, 1989, pp. 253-262.

doi:10.1007/978-3-642-83584-1 21

[21] Casalis, G., and Arnal, D., "ELFIN II Subtask 2.3: Database Method: Development and Validation of the Simplified Method for Pure Crossflow Instability at Low Speed," European Laminar Flow Investigation II TR 145, 1996.

[22] Vermeersch, O., and Bouteiller, X., "Numerical Study of Laminar Nacelles: Natural and Hybrid Laminar Flow Designs," International Journal of Engineering Systems Modelling and Simulation, Vol. 6, Nos. 3-4, 2014, pp. 191-204.

doi:10.1504/IJESMS.2014.063124

[23] Perraud, J., Arnal, D., Casalis, G., Archambaud, J. P., and Donelli, R., "Automatic Transition Predictions Using Simplified Methods," AIAA Journal, Vol. 47, No. 11, 2009, pp. 2676-2684. doi: $10.2514 / 1.42990$

[24] Vermeersch, O., Yoshida, K., Ueda, Y., and Arnal, D., "Natural Laminar Flow Wing for Supersonic Conditions: Wind Tunnel Experiments, Flight Test and Stability Computations," Progress in Aerospace Sciences, Vol. 79, Nov. 2015, pp. 64-91. doi:10.1016/j.paerosci.2015.07.003

[25] Bégou, G., Deniau, H., Vermeersch, O., and Casalis, G., "Database Approach for 2D Flow Transition Prediction in a RANS Code," 46th AIAA Fluid Dynamics Conference, AIAA Paper 2016-3488, June 2016 doi: $10.2514 / 6.2016-3488$

[26] Arnal, D., Habiballah, M., and Coustols, E., "Laminar Instability Theory and Transition Criteria in Two and Three-Dimensional Flow," La Recherche Aérospatiale (English Edition), No. 2, 1984, pp. 45-63.

[27] Cliquet, J., Houdeville, R., and Arnal, D., "Application of Laminar-Turbulent Transition Criteria in Navier-Stokes Computations," AIAA Journal, Vol. 46, No. 5, 2008, pp. 1182-1190. doi: $10.2514 / 1.30215$

[28] Arnal, D., Houdeville, R., Séraudie, A., and Vermeersch, O., "Overview of Laminar-Turbulent Transition Investigations at ONERA Toulouse," 41st AIAA Fluid Dynamics Conference and Exhibit, AIAA Paper 2011-3074, June 2011. doi: $10.2514 / 6.2011-3074$

[29] Gleyzes, C., Cousteix, J., and Bonnet, J. L., "Theoretical and Experimental Study of Low Reynolds Number Transitional Separation Bubbles," Conference on Low Reynolds Number Airfoil Aerodynamics, 1985.
[30] Beasley, J., "Calculation of the Laminar Boundary Layer and Prediction of Transition on a Sheared Wing," Reports and Memoranda of the Aeronautical Research Council, 3787, 1976.

[31] Hue, D., Péron, S., Wiart, L., Atinault, O., Gournay, E., Raud, P., Benoit, C., and Mayeur, J., "Validation of a Near-Body and Off-Body Grid Partitioning Methodology for Aircraft Aerodynamic Performance Prediction," Computers \& Fluids, Vol. 117, Aug. 2015, pp. 196-211. doi:10.1016/i.compfluid.2015.05.021

[32] Anon., "Mesh Generation Software for CFD—Pointwise," 2016, http:// www.pointwise.com [retrieved Oct. 2016].

[33] Cambier, L., Heib, S., and Plot, S., "The ONERA elsA CFD Software: Input from Research and Feedback from Industry," Mechanics and Industry, Vol. 14, No. 3, 2013, pp. 159-174. doi: $10.1051 / \mathrm{meca} / 2013056$

[34] Jameson, A., Schmidt, W., and Turkel, E., "Numerical Solution of the Euler Equations by Finite Volume Methods Using Runge Kutta Time Stepping Schemes," 14th Fluid and Plasma Dynamics Conference, AIAA Paper 1981-1259, June 1981. doi:10.2514/6.1981-1259

[35] Spalart, P. R., and Allmaras, S. R., "A One-Equation Turbulence Model for Aerodynamic Flows," 30th Aerospace Sciences Meeting and Exhibit, AIAA Paper 1992-0439, Jan. 1992. doi:10.2514/6.1992-439

[36] Spalart, P. R., "Strategies for Turbulence Modelling and Simulation," International Journal of Heat and Fluid Flow, Vol. 21, No. 3, 2000, pp. 252-263. doi:10.1016/S0142-727X(00)00007-2

[37] Hue, D., Chanzy, Q., and Landier, S., "DPW-6: Drag Analyses and Increments Using Different Geometries of the Common Research Model Airliner," Journal of Aircraft, Jan. 2017 doi:10.2514/1.C034139

[38] Destarac, D., "Far-Field/Near-Field Drag Balance Applications of Drag Extraction in CFD," CFD-Based Aircraft Drag Prediction and Reduction, VKI Lecture Series 2003-02, von Karman Inst. for Fluid Dynamics, Rhode-Saint-Genèse, Belgium, Nov. 2003.

[39] Hue, D., and Esquieu, S., "Computational Drag Prediction of the DPW4 Configuration Using the Far-Field Approach," Journal of Aircraft, Vol. 48, No. 5, Sept.-Oct. 2011, pp. 1658-1670. doi: $10.2514 / 1 . C 031337$ 